Research Article

\title{
Design of Head-Pursuit Guidance Law Based on Backstepping Sliding Mode Control
}

\author{
Chenqi Zhu $(\mathbb{D})$ and Zhengyu Guo \\ Northwestern Polytechnical University, Xi'an 710072, China \\ Correspondence should be addressed to Chenqi Zhu; 15036501865@163.com
}

Received 10 July 2019; Revised 2 October 2019; Accepted 16 October 2019; Published 3 December 2019

Academic Editor: Jacopo Serafini

Copyright (c) 2019 Chenqi Zhu and Zhengyu Guo. This is an open access article distributed under the Creative Commons Attribution License, which permits unrestricted use, distribution, and reproduction in any medium, provided the original work is properly cited.

\begin{abstract}
In order to meet the needs of high-precision guidance for intercepting hypersonic targets, a novel head-pursuit guidance law considering the dynamic characteristics of a missile control system and the target mobility is presented via combining a fast power reaching law with backstepping sliding mode control in this paper. Initially, a three-dimensional head-pursuit system model of the missile and target is established. Subsequently, the system model is decomposed into a pitch plane system and lateral plane system, the control system dynamics are equivalent to second-order systems, and finite-time disturbance observers are introduced to estimate the target accelerations. On the basis of the previous work, the head-pursuit guidance laws of the vertical system and the lateral system which can stabilize the closed-loop system are designed separately and strict proofs of the methods are given. Finally, simulations are carried out to verify the effectiveness of this head-pursuit guidance law.
\end{abstract}

\section{Introduction}

Hypersonic vehicles have the characteristics of long flight distance, high flight speed, and high maneuverability, which has great military value and also presents great challenges to the interception of such aircraft. As the first condition to affect the interception accuracy, the guidance law is one of the most important things in the design of the antiproximity space defense system [1-3]. Traditionally, there are two ways to intercept the targets, namely, tail-chase interception and head-on interception. Tail-chase interception requires the interceptor's speed to be higher than the target's, but it is hard to achieve when the target is a hypersonic vehicle. Head-on interception makes the missile fly to the target from the front of the target; the relative speed of the missile and target is so large that the response time of the interceptor will be shortened seriously, and this will put forward great challenges to the overload capability of the missile and the guidance law.
In order to solve the problems above, literatures $[4,5]$ put forward the head-pursuit interception for the first time; they proposed the conditions which must be met for head-pursuit interception and also designed a two-dimensional headpursuit guidance law under the ideal condition of ignoring the dynamic characteristics of autopilot. The head-pursuit guidance law not only reduced the requirement of overload capacity but also avoided the problem of aerodynamic ablation of interceptor warhead cover. On this basis, literature [6] designed the head-pursuit guidance law based on sliding mode control theory and verified the advantages of this method. Similarly, combined with the optimization theory, head-pursuit guidance laws based on Zero-Effort Miss (ZEM) and energy optimization are designed, respectively, in literatures $[7,8]$. However, the guidance laws designed above only consider the two-dimensional case, which is quite different from the actual interception.

Therefore, literatures $[9,10]$ designed three-dimensional head-pursuit guidance laws based on sliding mode control 


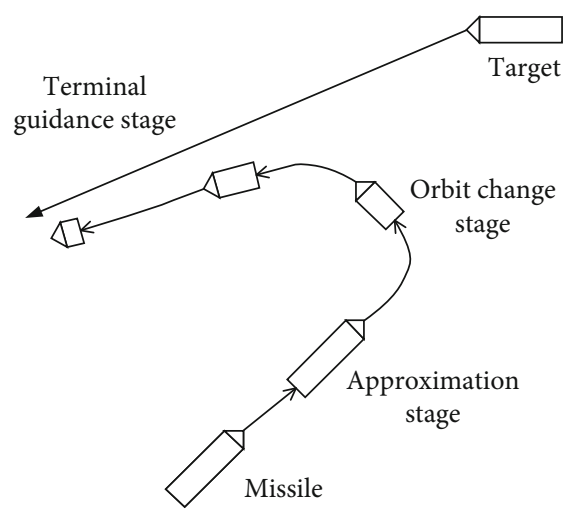

FIgURE 1: Head-pursuit interception schematic.

severally via considering the coupling between the pitch channel and the lateral channel. Besides, regarding the coupling between the pitch channel and the lateral channel as uncertain interference, adaptive sliding mode guidance laws of the pitch channel and the lateral channel are designed, respectively, in literature [11], which has strong robustness to the target acceleration; at the same time, a smooth second-order sliding mode guidance law which can effectively solve the discontinuous control problem caused by the target unknown acceleration and weaken the chattering is presented, too. In addition, adaptive sliding mode guidance laws are designed by Si and Song [12] to intercept the hypersonic vehicle in the form of head-pursuit interception. But these guidance laws ignore the dynamic characteristics of the control system. Even though literatures $[13,14]$ designed two-dimensional and three-dimensional head-pursuit guidance laws, respectively, they regard target maneuver as the precise known information, which is difficult to obtain accurately.

In order to meet the actual requirements of intercepting hypersonic vehicles, this paper considers the dynamic characteristics of the control system, estimates the unknown target maneuver with a finite-time disturbance observer (FTDO), and designs three-dimensional head-pursuit guidance laws based on the backstepping sliding mode control theory. Meanwhile, the fast reaching law is introduced to weaken chattering while ensuring the convergence speed of the closed-loop system.

The rest of this paper is organized as follows. Firstly, the principle of head-pursuit interception is introduced, and a three-dimensional head-pursuit system model of the missile and target is established. Secondly, the target maneuver is estimated by the FTDO and head-pursuit guidance laws which consider that the dynamic characteristics of the control system are designed and the stability of the closed-loop system is verified. Finally, the effectiveness and superiorities of the guidance laws designed in this paper are verified through the digital simulations.

\section{Problem Formulation}

As shown in Figure 1, the head-pursuit interception against the hypersonic vehicle can be divided into three stages:

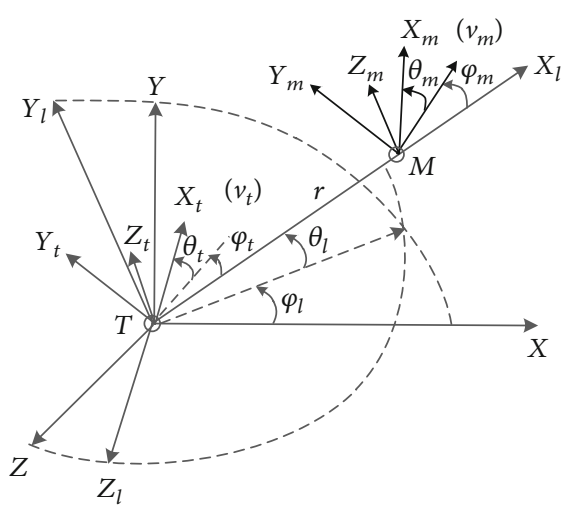

FIgURE 2: Three-dimensional engagement geometry.

approximation stage, orbit change stage, and terminal guidance stage. First, the missile approaches the target head-on after detecting it. Then, in order to make the interceptor consistent with the target's flight direction and reach the expected orbit, the rail control engine of the interceptor is used to change the trajectory at the predetermined time and position. Finally, the interceptor enters the terminal guidance stage, and the guidance law adjusts the trajectory to make the target collide with the interceptor from the back.

For the sake of simplicity, the missile and target are assumed to be point masses, and supposing that the acceleration just changes the direction of the velocity, the target acceleration and its rate of change are bounded. Now consider a three-dimensional interceptor and target engagement as shown in Figure 2.

In Figure 2, $T$ is the target, $M$ is the missile, $T-X Y Z$ is the inertial coordinate system (ICS), $T-X_{t} Y_{t} Z_{t}$ is the target velocity coordinate system (TVCS), $T-X_{m} Y_{m} Z_{m}$ is the missile velocity coordinate system (MVCS), $T-X_{l} Y_{l} Z_{l}$ is the line-of-sight coordinate system (LCS), $\varphi_{l}$ and $\theta_{l}$ are the direction angles of the LCS relative to the ICS, and $\varphi_{m}, \theta_{m}$ and $\varphi_{t}, \theta_{t}$ are the direction angles of the missile and target velocity vectors relative to the LCS, respectively. The moment when the missile enters the terminal guidance stage is defined as the initial moment, and the target position at this moment is taken as the coordinate origin to establish the ICS so as to obtain the three-dimensional relative motion model of headpursuit interception in the form of Equations (1), (2), (3), (4), (5), (6) and (7).

$$
\begin{aligned}
\dot{r} & =v_{m} \cos \theta_{m} \cos \varphi_{m}-v_{t} \cos \theta_{t} \cos \varphi_{t}, \\
\dot{\theta}_{l} & =\frac{v_{m} \sin \theta_{m}-v_{t} \sin \theta_{t}}{r}, \\
\dot{\varphi}_{l} & =\frac{v_{m} \cos \theta_{m} \sin \varphi_{m}-v_{t} \cos \theta_{t} \sin \varphi_{t}}{r \cos \theta_{l}},
\end{aligned}
$$

$$
\dot{\theta}_{t}=\frac{a_{y t}}{v_{t}}-\dot{\varphi}_{l} \sin \theta_{l} \sin \varphi_{t}-\dot{\theta}_{l} \cos \varphi_{t},
$$




$$
\begin{aligned}
\dot{\varphi}_{t}= & -\frac{a_{z t}}{v_{t} \cos \theta_{t}}+\dot{\varphi}_{l} \sin \theta_{l} \cos \varphi_{t} \tan \theta_{t} \\
& -\dot{\theta}_{l} \sin \varphi_{t} \tan \theta_{t}-\dot{\varphi}_{l} \cos \theta_{l}, \\
\dot{\theta}_{m}= & \frac{a_{y m}}{v_{m}}-\dot{\varphi}_{l} \sin \theta_{l} \sin \varphi_{m}-\dot{\theta}_{l} \cos \varphi_{m}, \\
\dot{\varphi}_{m}= & -\frac{a_{z m}}{v_{m} \cos \theta_{m}}+\dot{\varphi}_{l} \sin \theta_{l} \cos \varphi_{m} \tan \theta_{m} \\
& -\dot{\theta}_{l} \sin \varphi_{m} \tan \theta_{m}-\dot{\varphi}_{l} \cos \theta_{l},
\end{aligned}
$$

where $a_{y t}, a_{z t}$ and $a_{y m}, a_{z m}$ are the components of the missile and target acceleration vectors in the vertical direction of their respective velocity directions and $v_{t}$ and $v_{m}$ are the magnitude of missile and target velocities, respectively. According to the guidance conditions of the head-pursuit interception discussed in literature [9], if the missile's variables meet Equations (8) and (12), the interceptor can achieve head-pursuit interception of the target:

$$
\begin{aligned}
\lim _{r \rightarrow 0} \varphi_{m} & =0 ; \\
\lim _{r \rightarrow 0} \varphi_{t} & =0 ; \\
\lim _{r \rightarrow 0} \theta_{m} & =0 ; \\
\lim _{r \rightarrow 0} \theta_{t} & =0, \\
\varphi_{m} & =n_{1} \varphi_{t} ; \\
\theta_{m} & =n_{2} \theta_{t},
\end{aligned}
$$

where $n_{1}$ and $n_{2}$ are the lead factors and need to meet the following conditions:

$$
\begin{aligned}
& n_{1}>\frac{v_{t}}{v_{m}}, \\
& n_{2}>\frac{v_{t}}{v_{m}} .
\end{aligned}
$$

It has been proved that in the head-pursuit interception, if the system shown in Equations (1), (2), (3), (4), (5), (6) and (7) meets the guidance relationship corresponding to
Equation (12), the target can be successfully intercepted. Therefore, the key of designing the head-pursuit guidance law is to design the controller to make the system satisfy Equation (12).

\section{Introduction to Basic Knowledge}

Literature [15] presented that the dynamic characteristics of the missile can be described by a second-order model approximately, which can be expressed as follows:

$$
\begin{aligned}
& \ddot{a}_{y m}=-2 \xi \omega_{n} \dot{a}_{y m}-\omega_{n}^{2} a_{y m}+\omega_{n}^{2} a_{y m}^{c}, \\
& \ddot{a}_{z m}=-2 \xi \omega_{n} \dot{a}_{z m}-\omega_{n}^{2} a_{z m}+\omega_{n}^{2} a_{z m}^{c},
\end{aligned}
$$

where $\xi$ is the damping coefficient, $\omega_{n}$ is oscillation frequency, and $a_{y m}^{c}$ and $a_{z m}^{c}$ are the acceleration instruction.

Lemma 1. [16]. Consider a nonlinear system in the form of $\dot{s}=-k_{1} s-k_{2} \operatorname{sig}^{\rho}(s)$, where $k_{1}, k_{2}>0, \rho \in(0,1)$. Then, $s$ and $\dot{s}$ stabilize within a limited time $T$ which depends on initial conditions and satisfies the following equality:

$$
T(s(0))=\frac{\ln \left(1+\left.k_{1}\left|k_{2}\right| s(0)\right|^{1-\rho}\right)}{k_{1}(1-\rho)} .
$$

Note 1. $k_{1}$ and $k_{2}$ reflect the speed of the system state approaching 0 ; when the system state is far from the stable state, $k_{1}$ plays a major role, but when the system state is near the stable state, $k_{2}$ plays a major role. The reaching law which has the same form as this system is defined as the fast power reaching law; the convergence speed of the system state can be accelerated, and the chattering can be weakened via selecting proper $k_{1}$ and $k_{2}$.

Lemma 2. [17]. Consider a nonlinear system in the form of $\dot{x}=g(t)+u(t), x \in R, u$ is a continuous control input and satisfies $u(t) \in R, g(t)$ is a smooth uncertain function and satisfies $|\dot{g}(t)| \leq L$. Then, there is a disturbance observer in the form of Equation (17), which makes the system satisfy Equation (18) after a transient process:

$$
\begin{aligned}
& \left\{\begin{array}{l}
\dot{z}_{0}=v_{0}+u(t), \quad v_{0}=-\lambda_{2} L^{1 / 3}\left|z_{0}-x_{1}\right|^{2 / 3} \operatorname{sign}\left(z_{0}-x_{1}\right)-\mu_{2}\left(z_{0}-x_{1}\right)+z_{1}, \\
\dot{z}_{1}=v_{1}, \quad v_{1}=-\lambda_{1} L^{1 / 2}\left|z_{1}-v_{0}\right|^{1 / 2} \operatorname{sign}\left(z_{1}-v_{0}\right)-\mu_{1}\left(z_{1}-v_{0}\right)+z_{2}, \\
\dot{z}_{2}=v_{2}, \quad v_{2}=-\lambda_{0} L \operatorname{sign}\left(z_{2}-v_{1}\right)-\mu_{0}\left(z_{2}-v_{1}\right),
\end{array}\right. \\
& z_{10}(t)=x_{11}(t), \\
& z_{11}(t)=d_{1}(t), \\
& z_{12}(t)=\dot{d}_{1}(t),
\end{aligned}
$$


where $\lambda_{0}, \lambda_{1}, \lambda_{2}$ and $\mu_{0}, \mu_{1}, \mu_{2}$ are the design parameters of the disturbance observer.

Note 2. Zhou [17] gives a valid set of values for $\lambda_{0}, \lambda_{1}, \lambda_{2}$ and $\mu_{0}, \mu_{1}, \mu_{2}$ as follows:

$$
\begin{aligned}
& \lambda_{0}=8, \\
& \lambda_{1}=5, \\
& \lambda_{2}=3, \\
& \mu_{0}=12, \\
& \mu_{1}=11, \\
& \mu_{2}=10 .
\end{aligned}
$$

3.1. Design of the Head-Pursuit Guidance Law. The design of SMG law can be divided into two phases which are the reaching phase (RP) and the sliding mode phase (SMP). Literature [4] has proved the convergence of SMP; therefore, only the convergence of the first stage is studied here.

Based on the previous description, the following variables are defined:

$$
\begin{aligned}
& x_{11}=\theta_{m}-n \theta_{t}, \\
& x_{12}=a_{y m}, \\
& x_{13}=\dot{a}_{y m}, \\
& x_{21}=\varphi_{m}-n \varphi_{t}, \\
& x_{22}=a_{z m}, \\
& x_{23}=\dot{a}_{z m}, \\
& u_{y}=a_{y m}^{c}, \\
& u_{z}=a_{z m}^{c} .
\end{aligned}
$$

Subsequently, the system model is decomposed into the pitch plane system in the form of Equation (21) and the lateral plane system in the form of Equation (22):

$$
\begin{aligned}
& \left\{\begin{array}{l}
\dot{x}_{11}=\frac{x_{12}}{v_{m}}+f_{1}-n f_{3}+d_{1}, \\
\dot{x}_{12}=x_{13}, \\
\dot{x}_{13}=-2 \xi \omega_{n} x_{13}-\omega_{n}^{2} x_{12}+\omega_{n}^{2} u_{y},
\end{array}\right. \\
& \left\{\begin{array}{l}
\dot{x}_{21}=-\frac{x_{22}}{v_{m} \cos \theta_{m}}+f_{2}-n f_{4}+d_{2}, \\
\dot{x}_{22}=x_{23}, \\
\dot{x}_{23}=-2 \xi \omega_{n} x_{23}-\omega_{n}^{2} x_{22}+\omega_{n}^{2} u_{z},
\end{array}\right.
\end{aligned}
$$

where

$f_{1}=-\dot{\varphi}_{l} \sin \theta_{l} \sin \varphi_{m}-\dot{\theta}_{l} \cos \varphi_{m}$

$f_{2}=\dot{\varphi}_{l} \sin \theta_{l} \cos \varphi_{m} \tan \theta_{m}-\dot{\theta}_{l} \sin \varphi_{m} \tan \theta_{m}-\dot{\varphi}_{l} \cos \theta_{l}$,

$f_{3}=-\dot{\varphi}_{l} \sin \theta_{l} \sin \varphi_{t}-\dot{\theta}_{l} \cos \varphi_{t}$,

$f_{4}=\dot{\varphi}_{l} \sin \theta_{l} \cos \varphi_{t} \tan \theta_{t}-\dot{\theta}_{l} \sin \varphi_{t} \tan \theta_{t}-\dot{\varphi}_{l} \cos \theta_{l}$,

$d_{1}=-n\left(\frac{a_{y t}}{v_{t}}\right)$

$d_{2}=n\left(\frac{a_{z t}}{v_{t} \cos \theta_{t}}\right)$.

3.1.1. Guidance Law Design for the Pitch Plane System. Firstly, define $L_{1}$ and $h_{1}$ as follows:

$$
\begin{aligned}
\left|\dot{d}_{1}(t)\right| & \leq L_{1}, \\
h_{1} & =\frac{x_{12}}{v_{m}}+f_{1}-n f_{3} .
\end{aligned}
$$

Secondly, a FTDO is designed to estimate the disturbance $d_{1}$ in the pitch plane system via combining Lemma 2 with the initial angle conditions of head-pursuit interception discussed by Ge et al. [10]:

$$
\left\{\begin{array}{l}
\dot{z}_{10}=v_{10}+h_{1}(t), \quad v_{10}=-\lambda_{12} L_{1}^{1 / 3}\left|z_{10}-x_{11}\right|^{2 / 3} \operatorname{sign}\left(z_{10}-x_{11}\right)-\mu_{12}\left(z_{10}-x_{11}\right)+z_{11}, \\
\dot{z}_{11}=v_{11}, \quad v_{11}=-\lambda_{11} L_{1}^{1 / 2}\left|z_{11}-v_{10}\right|^{1 / 2} \operatorname{sign}\left(z_{11}-v_{10}\right)-\mu_{11}\left(z_{11}-v_{10}\right)+z_{12} \\
\dot{z}_{12}=v_{12}, \quad v_{12}=-\lambda_{10} L_{1} \operatorname{sign}\left(z_{12}-v_{11}\right)-\mu_{10}\left(z_{12}-v_{11}\right)
\end{array}\right.
$$

where $\lambda_{10}, \lambda_{11}, \lambda_{12}$ and $\mu_{10}, \mu_{11}, \mu_{12}$ are the design parameters of the disturbance observer.

After a transient process of finite time, the following equation can be obtained:

$$
z_{10}(t)=x_{11}(t)
$$

$$
\begin{gathered}
z_{11}(t)=d_{1}(t), \\
z_{12}(t)=\dot{d}_{1}(t) .
\end{gathered}
$$

Then, guidance based on backstepping sliding mode control theory is designed according to the following steps. 
(1) The $1^{\text {st }}$ dynamic error surface is defined as

$$
s_{11}=x_{11}
$$

Taking the derivative of $s_{11}$ and obtaining the error dynamic equation

$$
\dot{s}_{11}=\frac{x_{12}}{v_{m}}+f_{1}-n f_{3}+d_{1} .
$$

Defining a fast power reaching law based on Lemma 1 as follows:

$$
\dot{s}_{11}=-k_{11} s_{11}-k_{12} \operatorname{sig}^{\rho_{11}}\left(s_{11}\right),
$$

where $k_{11}>0, k_{12}>0$, and $0<\rho_{11}<1$. Substitute Equation (26) into Equation (30) and combine Equation (30) with Equation (31), the following virtual control input of the $1^{\text {st }}$ dynamic surface can be obtained:

$$
x_{12}^{c}=-v_{m}\left[k_{11} s_{11}+k_{12} \operatorname{sig}^{\rho_{11}}\left(s_{11}\right)+f_{1}-n f_{3}+z_{11}\right] .
$$

A $1^{\text {st }}$-order low-pass filter is designed in the following form for $x_{12}^{c}$ to avoid the increase of calculating complexity caused by the "explosion of terms" while finding the derivative of the virtual control input:

$$
\begin{aligned}
\dot{x}_{12}^{c *} & =\tau_{11}^{-1}\left(x_{12}^{c}-x_{12}^{c *}\right), \\
x_{12}^{c *}(0) & =x_{12}^{c}(0),
\end{aligned}
$$

where $x_{12}^{c *}$ is filtered virtual control input and $\tau_{11}$ is the time constant of the filter

(2) The $2^{\text {nd }}$ dynamic error surface is defined as

$$
s_{12}=x_{12}-x_{12}^{c *}
$$

Taking the derivative of $s_{12}$ and obtaining the error dynamic equation

$$
\dot{s}_{12}=x_{13}-\dot{x}_{12}^{c *}
$$

Defining a fast power reaching law as follows:

$$
\dot{s}_{12}=-k_{13} s_{12}-k_{14} \operatorname{sig}^{\rho_{12}}\left(s_{12}\right),
$$

where $k_{13}>0, k_{14}>0$, and $0<\rho_{12}<1$. Combine Equation (35) with Equation (36), the following virtual control input of the $2^{\text {nd }}$ dynamic surface can be obtained:

$$
x_{13}^{c}=-k_{13} s_{12}-k_{14} \operatorname{sig}^{\rho_{12}}\left(s_{12}\right)+\dot{x}_{12}^{c *} \text {. }
$$

Similarly, $x_{13}^{c}$ is fed through a low-pass filter in the following form to obtain the filtered virtual control input:

$$
\begin{aligned}
\dot{x}_{13}^{c *} & =\tau_{12}^{-1}\left(x_{13}^{c}-x_{13}^{c *}\right), \\
x_{13}^{c *}(0) & =x_{13}^{c}(0),
\end{aligned}
$$

where $x_{13}^{c *}$ is filtered virtual control input and $\tau_{12}$ is the time constant of the filter

(3) The $3^{\text {rd }}$ dynamic error surface is defined as

$$
s_{13}=x_{13}-x_{13}^{c *} \text {. }
$$

Taking the derivative of $s_{13}$ and obtaining the error dynamic equation

$$
\dot{s}_{13}=\dot{x}_{13}-\dot{x}_{13}^{c *}=-2 \xi \omega_{n} x_{13}-\omega_{n}^{2} x_{12}+\omega_{n}^{2} u_{y}-\dot{x}_{13}^{c *} .
$$

Defining a fast power reaching law as follows:

$$
\dot{s}_{13}=-k_{15} s_{13}-k_{16} \operatorname{sig}^{\rho_{13}}\left(s_{13}\right)
$$

where $k_{15}>0, k_{16}>0$, and $0<\rho_{13}<1$. Combine Equation (40) with Equation (41), the following control input of the $3^{\text {rd }}$ dynamic surface can be obtained:

$$
u_{y}=\omega_{n}^{-2}\left[-k_{15} s_{13}-k_{16} \operatorname{sig}^{\rho_{13}}\left(s_{13}\right)+2 \xi \omega_{n} x_{13}+\omega_{n}^{2} x_{12}+\dot{x}_{13}^{c *}\right] .
$$

Therefore, the head-pursuit guidance law for the pitch plane system has the following form:

$$
\left\{\begin{array}{l}
s_{11}=x_{11}, \\
x_{12}^{c}=-v_{m}\left[k_{11} s_{11}+k_{12} \operatorname{sig}^{\rho_{11}}\left(s_{11}\right)+f_{1}-n f_{3}+z_{11}\right], \\
\dot{x}_{12}^{c *}=\tau_{11}^{-1}\left(x_{12}^{c}-x_{12}^{c *}\right), \quad x_{12}^{c *}(0)=x_{12}^{c}(0), \\
s_{12}=x_{12}-x_{12}^{c *}, \\
x_{13}^{c}=-k_{13} s_{12}-k_{14} \operatorname{sig}^{\rho_{12}}\left(s_{12}\right)+\dot{x}_{12}^{c *}, \\
\dot{x}_{13}^{c *}=\tau_{12}^{-1}\left(x_{13}^{c}-x_{13}^{c *}\right), \quad x_{13}^{c *}(0)=x_{13}^{c}(0), \\
s_{13}=x_{13}-x_{13}^{c *}, \\
u_{y}=\omega_{n}^{-2}\left[-k_{15} s_{13}-k_{16} \operatorname{sig}^{\rho_{13}}\left(s_{13}\right)+2 \xi \omega_{n} x_{13}+\omega_{n}^{2} x_{12}+\dot{x}_{13}^{c *}\right] .
\end{array}\right.
$$

Finally, the stability of the guidance law in the form of Equation (43) is analyzed in the coming section.

Defining the Lyapunov function as follows:

$$
V_{1}=\frac{1}{2} s_{11}^{2}+\frac{1}{2} s_{12}^{2}+\frac{1}{2} s_{13}^{2}+\frac{1}{2} e_{13}^{2}+\frac{1}{2} e_{14}^{2},
$$


where

$$
\begin{aligned}
& e_{13}=x_{12}^{c *}-x_{12}^{c}, \\
& e_{14}=x_{13}^{c *}-x_{13}^{c} .
\end{aligned}
$$

According to Equations (21) and (43), the following equations can be obtained:

$$
\begin{aligned}
\dot{s}_{11} & =\frac{x_{12}}{v_{m}}+f_{1}-n f_{3}+d_{1}=\frac{s_{12}+e_{13}+x_{12}^{c}}{v_{m}}+f_{1}-n f_{3}+d_{1} \\
& =\frac{s_{12}+e_{13}}{v_{m}}-\left[k_{11} s_{11}+k_{12} \operatorname{sig}^{\rho_{11}}\left(s_{11}\right)\right], \\
\dot{s}_{12} & =x_{13}-\dot{x}_{12}^{c *}=s_{13}+x_{13}^{c *}-\dot{x}_{12}^{c *}=s_{13}+e_{14}+x_{13}^{c}-\dot{x}_{12}^{c *} \\
& =s_{13}+e_{14}-k_{13} s_{12}-k_{14} \operatorname{sig}^{\rho_{12}}\left(s_{12}\right), \\
\dot{s}_{13} & =-2 \xi \omega_{n} x_{13}-\omega_{n}^{2} x_{12}+\omega_{n}^{2} u_{y}-\dot{x}_{13}^{c *} \\
& =-k_{15} s_{13}-k_{16} \operatorname{sig}^{\rho_{13}}\left(s_{13}\right),
\end{aligned}
$$

where

$$
e_{12}=d_{1}-z_{11}
$$

Then,

$$
\begin{aligned}
s_{11} \dot{s}_{11}= & s_{11}\left\{\frac{s_{12}+e_{13}}{v_{m}}-\left[k_{11} s_{11}+k_{12} \operatorname{sig}^{\rho_{11}}\left(s_{11}\right)\right]\right\} \\
\leq & \frac{1}{2 v_{m}}\left(s_{11}^{2}+s_{12}^{2}\right)+\frac{1}{2 v_{m}}\left(s_{11}^{2}+e_{13}^{2}\right)-k_{11} s_{11}^{2} \\
& +k_{12}\left(\frac{1}{2}+\frac{3}{2} s_{11}^{2}\right)=\left(\frac{1}{v_{m}}-k_{11}+\frac{3}{2} k_{12}\right) s_{11}^{2} \\
& +\frac{1}{2 v_{m}} s_{12}^{2}+\frac{1}{2 v_{m}} e_{13}^{2}+\frac{1}{2} k_{12}, \\
s_{12} \dot{s}_{12}= & s_{12}\left[s_{13}+e_{14}-k_{13} s_{12}-k_{14} \operatorname{sig}^{\rho_{12}}\left(s_{12}\right)\right] \\
\leq & \frac{1}{2}\left(s_{12}^{2}+s_{13}^{2}+s_{12}^{2}+e_{14}^{2}\right)-k_{13} s_{12}^{2}+k_{14}\left(\frac{1}{2}+\frac{3}{2} s_{12}^{2}\right) \\
= & \left(1-k_{13}+\frac{3}{2} k_{14}\right) s_{12}^{2}+\frac{1}{2} s_{13}^{2}+\frac{1}{2} e_{14}^{2}+\frac{1}{2} k_{14}, \\
s_{13} \dot{s}_{13}= & -k_{15} s_{13}^{2}-k_{16}\left|s_{13}\right|_{13}^{\rho_{13}+1} \leq-k_{15} s_{13}^{2}+k_{16}\left(\frac{1}{2}+\frac{3}{2} s_{13}^{2}\right) \\
= & \left(-k_{15}+\frac{3}{2} k_{16}\right) s_{13}^{2}+\frac{1}{2} k_{16}, \\
e_{14} \dot{e}_{14}= & \left.e_{14}\left(-\tau_{12}^{-1} e_{14}-\dot{x}_{13}^{c}\right) \leq-\tau_{12}^{-1} e_{14}^{2}+\frac{1}{2} e_{14}^{2}+\frac{1}{2}\left|\dot{x}_{13}^{c}\right|^{2}\right) \\
= & \left(-\tau_{12}^{-1}+\frac{1}{2}\right) e_{14}^{2} \frac{1}{2}\left|\dot{x}_{13}^{c}\right|^{2} . \\
e_{13} \dot{e}_{13}= & e_{13}\left(-\tau_{11}^{-1} e_{13}-\dot{x}_{12}^{c}\right) \leq-\tau_{11}^{-1} e_{13}^{2}+\frac{1}{2} e_{13}^{2}+\frac{1}{2}\left|\dot{x}_{12}^{c}\right|^{2} \\
= & \left(-\tau_{11}^{-1}+\frac{1}{2}\right) e_{13}^{2} \frac{1}{2}\left|\dot{x}_{12}^{c}\right|^{2},
\end{aligned}
$$

Taking the derivative of Equation (44):

$$
\begin{aligned}
\dot{V}_{1}= & s_{11} \dot{s}_{11}+s_{12} \dot{s}_{12}+s_{13} \dot{s}_{13}+e_{13} \dot{e}_{13}+e_{14} \dot{e}_{14} \\
\leq & \left(\frac{1}{v_{m}}-k_{11}+\frac{3}{2} k_{12}\right) s_{11}^{2}+\left(\frac{1}{2 v_{m}}+1-k_{13}+\frac{3}{2} k_{14}\right) s_{12}^{2} \\
& +\left(\frac{1}{2}-k_{15}+\frac{3}{2} k_{16}\right) s_{13}^{2}+\left(-\tau_{11}^{-1}+\frac{1}{2}+\frac{1}{2 v_{m}}\right) e_{13}^{2} \\
& +\left(-\tau_{12}^{-1}+1\right) e_{14}^{2}+\frac{1}{2}\left|\dot{x}_{12}^{c}\right|^{2}+\frac{1}{2}\left|\dot{x}_{13}^{c}\right|^{2}+\frac{1}{2} k_{12} \\
& +\frac{1}{2} k_{14}+\frac{1}{2} k_{16} .
\end{aligned}
$$

Since all variables and their derivatives in the system are bounded, there exist nonnegative continuous functions $g_{1}$ and $g_{2}$ as follows:

$$
\left\{\begin{array}{l}
\left|\dot{x}_{12}^{c *}\right| \leq g_{1}\left(s_{11}, s_{12}, e_{12}, e_{13}, k_{11}, k_{12}\right), \\
\left|\dot{x}_{13}^{c *}\right| \leq g_{2}\left(s_{11}, s_{12}, s_{13}, e_{12}, e_{13}, e_{14}, k_{11}, k_{12}, k_{13}, k_{14}\right) .
\end{array}\right.
$$

There are positive constant $R_{1}$ and compact set $B_{1}$ which satisfy the following equation:

$$
B_{1}=\left\{\left[s_{11}, s_{12}, s_{13}, e_{12}, e_{13}, e_{14}\right]^{T}, V_{1}<R_{1}\right\} .
$$

Therefore, we have

$$
\left\{\begin{array}{l}
\left|\dot{x}_{12}^{c}\right| \leq M_{11} \\
\left|\dot{x}_{13}^{c}\right| \leq M_{12}
\end{array}\right.
$$

where $M_{11}$ and $M_{12}$ are the maximum values of $g_{1}$ and $g_{2}$ on the compact set $B_{1}$, respectively. Rewrite Equation (49), and we get

$$
\begin{aligned}
\dot{V}_{1} \leq & \left(\frac{1}{v_{m}}-k_{11}+\frac{3}{2} k_{12}\right) s_{11}^{2}+\left(\frac{1}{2 v_{m}}+1-k_{13}+\frac{3}{2} k_{14}\right) s_{12}^{2} \\
& +\left(\frac{1}{2}-k_{15}+\frac{3}{2} k_{16}\right) s_{13}^{2}+\left(-\tau_{11}^{-1}+\frac{1}{2}+\frac{1}{2 v_{m}}\right) e_{13}^{2} \\
& +\left(-\tau_{12}^{-1}+1\right) e_{14}^{2}+\frac{1}{2} M_{11}^{2}+\frac{1}{2} M_{12}^{2} \\
& +\frac{1}{2} k_{12}+\frac{1}{2} k_{14}+\frac{1}{2} k_{16} .
\end{aligned}
$$

Let $N$ be a positive integer. If design parameters satisfy the following equation: 


$$
\left\{\begin{array}{l}
k_{11}-\frac{3}{2} k_{12} \geq \frac{1}{v_{m}}+0.5 N \\
k_{13}-\frac{3}{2} k_{14} \geq \frac{1}{2 v_{m}}+1+0.5 N \\
k_{15}-\frac{3}{2} k_{16} \geq \frac{1}{2}+0.5 N \\
\tau_{11}^{-1} \geq \frac{1}{2}+\frac{1}{2 v_{m}}+0.5 N \\
\tau_{12}^{-1} \geq 1+0.5 N
\end{array}\right.
$$

We get the following inequality:

$$
\dot{V}_{1} \leq-N V+E
$$

where

$$
E=\frac{1}{2} M_{11}^{2}+\frac{1}{2} M_{12}^{2}+\frac{1}{2} k_{12}+\frac{1}{2} k_{14}+\frac{1}{2} k_{16} .
$$

$$
\begin{aligned}
& \text { According to Equation (55), we can get } \\
& \qquad V_{1} \leq \frac{[N V(0)-E] e^{-N t}+E}{N} .
\end{aligned}
$$

Based on the literature proposed by Liu and Liang [18], if $N$ is large enough, it is guaranteed that filtering errors and error surfaces are sufficiently small. Therefore, just make sure that the design parameters $k_{11}, k_{13}, k_{15}$ are large enough; the design parameters $k_{12}, k_{14}, k_{16}$ and the time constant of the filter $\tau_{11}, \tau_{12}$ are small enough.

3.1.2. Guidance Law Design for the Lateral Plane System. The design of the guidance law for the lateral plane system is similar to the pitch plane system. Firstly, a FTDO is designed to estimate the disturbance $d_{2}$ in the lateral plane system:

$$
\left\{\begin{array}{l}
\dot{z}_{20}=v_{20}+h_{2}(t), \quad v_{20}=-\lambda_{22} L_{2}^{1 / 3}\left|z_{20}-x_{21}\right|^{2 / 3} \operatorname{sign}\left(z_{20}-x_{21}\right)-\mu_{22}\left(z_{20}-x_{21}\right)+z_{21} \\
\dot{z}_{21}=v_{21}, \quad v_{21}=-\lambda_{21} L_{2}^{1 / 2}\left|z_{21}-v_{20}\right|^{1 / 2} \operatorname{sign}\left(z_{21}-v_{20}\right)-\mu_{21}\left(z_{21}-v_{20}\right)+z_{22} \\
\dot{z}_{22}=v_{22}, \quad v_{22}=-\lambda_{20} L_{2} \operatorname{sign}\left(z_{22}-v_{21}\right)-\mu_{20}\left(z_{22}-v_{21}\right)
\end{array}\right.
$$

where $\lambda_{20}, \lambda_{21}, \lambda_{22}$ and $\mu_{20}, \mu_{21}, \mu_{22}$ are the design parameters of the disturbance observer, $z_{20}, z_{21}, z_{22}$ are corresponding estimates, and $L_{2}$ and $h_{2}$ satisfy the following relations, respectively:

$$
\begin{gathered}
L_{2} \geq\left|\dot{d}_{2}(t)\right| \\
h_{2}=-\frac{x_{22}}{v_{m} \cos \theta_{m}}+f_{2}-n f_{4} .
\end{gathered}
$$

The head-pursuit guidance law for the lateral plane system is designed as follows:

$$
\left\{\begin{array}{l}
s_{21}=x_{21}, \\
x_{22}^{c}=v_{m} \cos \theta_{m}\left[k_{21} s_{21}+k_{22} \operatorname{sig}^{\rho_{21}}\left(s_{21}\right)+f_{2}-n f_{4}+z_{21}\right] \\
\dot{x}_{22}^{c *}=\tau_{21}^{-1}\left(x_{22}^{c}-x_{22}^{c *}\right), \quad x_{22}^{c *}(0)=x_{22}^{c}(0) \\
s_{22}=x_{22}-x_{22}^{c *}, \\
x_{23}^{c}=-k_{23} s_{22}-k_{24} \operatorname{sig}^{\rho_{22}}\left(s_{22}\right)+\dot{x}_{22}^{c *}, \\
\dot{x}_{23}^{c *}=\tau_{22}^{-1}\left(x_{23}^{c}-x_{23}^{c *}\right), \quad x_{23}^{c *}(0)=x_{23}^{c}(0) \\
s_{23}=x_{23}-x_{23}^{c *}, \\
u_{z}=\omega_{n}^{-2}\left[-k_{25} s_{23}-k_{26} \operatorname{sig}^{\rho_{23}}\left(s_{23}\right)+2 \xi \omega_{n} x_{23}+\omega_{n}^{2} x_{22}+\dot{x}_{23}^{c *}\right]
\end{array}\right.
$$

where $s_{21}, s_{22}, s_{23}$ are the corresponding error surfaces, $k_{21}$, $k_{22}, k_{23}, k_{24}, k_{25}, k_{26}$ are the corresponding design parameters, $x_{22}^{c}, x_{23}^{c}, x_{22}^{c *}, x_{23}^{c *}$ are the corresponding virtual control inputs and filtered virtual control inputs, and $\tau_{21}, \tau_{22}$ are the corresponding time constants of the filters. If the design parameters $k_{21}, k_{23}, k_{25}$ are large enough, the design parameters $k_{22}, k_{24}, k_{26}$ and the time constant of the filter $\tau_{21}, \tau_{22}$ are small enough; it can be ensured that filtering errors and error surfaces are sufficiently small.

The stability of the guidance law for the lateral plane system is similar to the pitch plane system; therefore, there is no specific elaboration here.

\section{Numerical Simulation}

In order to verify the effectiveness of the guidance law designed in this paper, the guidance laws based on the power reaching law and exponential approach law are selected for comparisons.

The guidance law based on the power reaching law is given as Equation (61), and the guidance law based on the exponential approach law is given as Equation (62):

$$
U_{1}=\left[\begin{array}{l}
u_{11} \\
u_{12}
\end{array}\right]=\left[\begin{array}{c}
-v_{m}\left[k_{31} \operatorname{sig}^{\rho_{31}}\left(s_{11}\right)+f_{1}-n f_{3}\right] \\
v_{m} \cos \theta_{m}\left(k_{32} \operatorname{sig}^{\rho_{32}}\left(s_{21}\right)+f_{2}-n f_{4}\right)
\end{array}\right],
$$




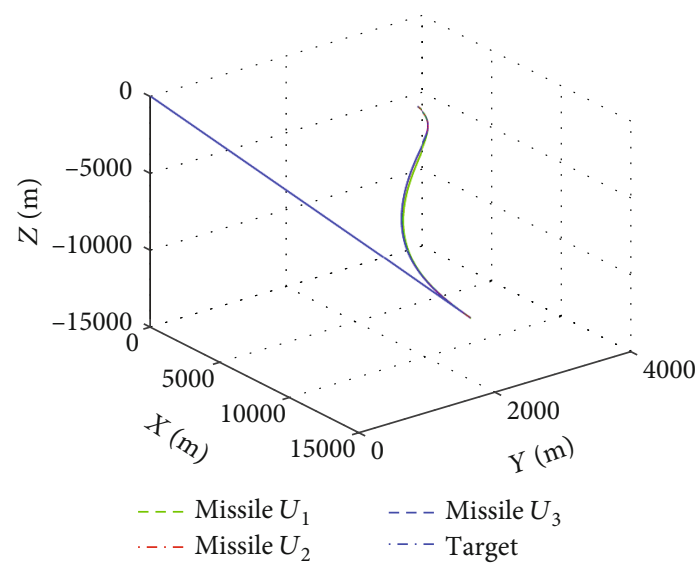

Figure 3: Relative trajectories.

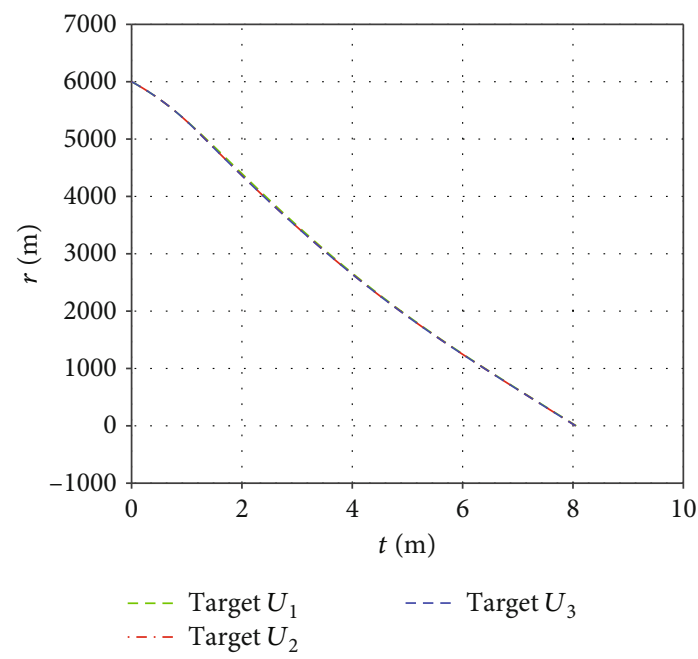

Figure 4: Ranges between the missile and the target.

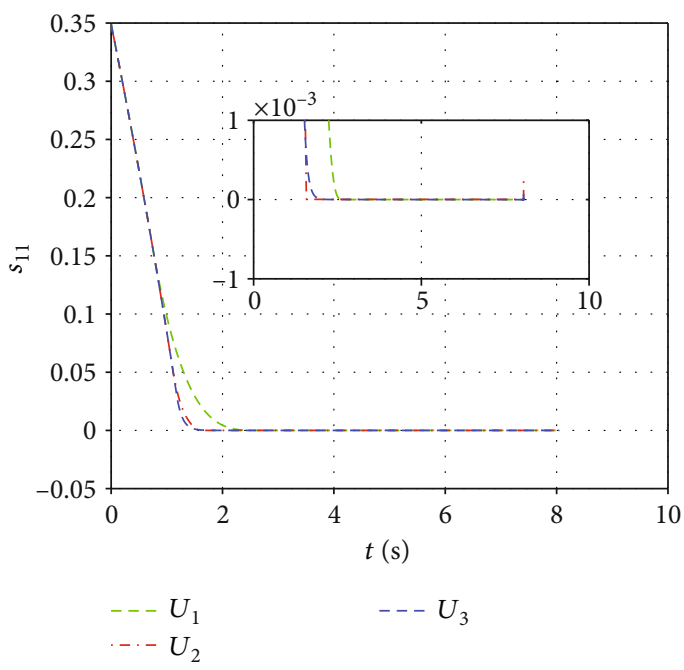

Figure 5: Dynamic error surfaces $s_{11}$.

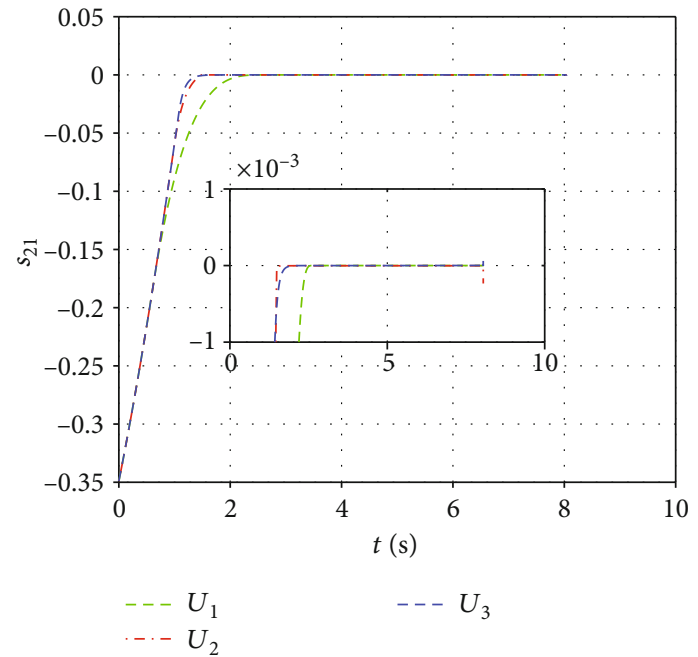

Figure 6: Dynamic error surfaces $s_{21}$.

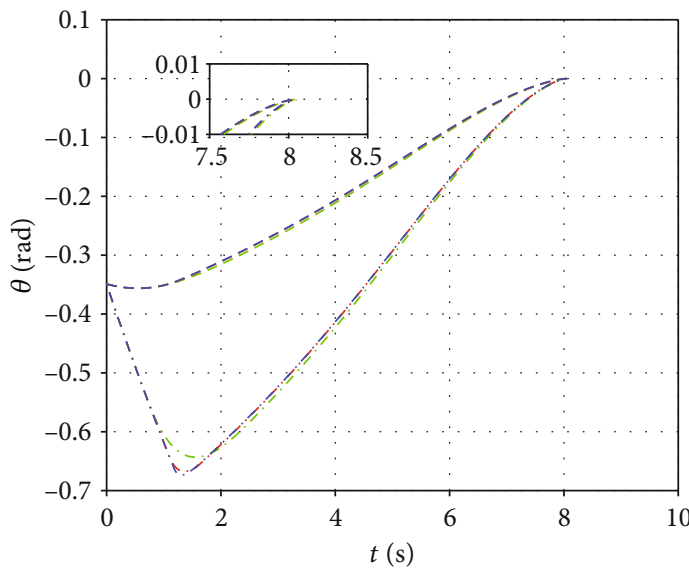

$$
\begin{array}{ll}
-\cdots \theta_{t}\left(U_{1}\right) & \ldots-\theta_{m}\left(U_{2}\right) \\
\cdots-\theta_{m}\left(U_{1}\right) & -\cdots \theta_{t}\left(U_{3}\right) \\
---\theta_{t}\left(U_{2}\right) & \cdots
\end{array}
$$

Figure 7: Direction angles $\theta$.

$$
U_{2}=\left[\begin{array}{l}
u_{21} \\
u_{22}
\end{array}\right]=\left[\begin{array}{c}
-v_{m}\left[k_{41} s_{11}+\sigma_{41} \operatorname{sign}\left(s_{11}\right)+f_{1}-n f_{3}\right] \\
v_{m} \cos \theta_{m}\left[k_{42} s_{21}+\sigma_{42} \operatorname{sign}\left(s_{21}\right)+f_{2}-n f_{4}\right]
\end{array}\right] .
$$

The guidance law proposed in this paper which considers the dynamic characteristics is rewritten as follows:

$$
U_{3}=\left[\begin{array}{l}
u_{y} \\
u_{z}
\end{array}\right]=\left[\begin{array}{l}
\omega_{n}^{-2}\left[-k_{15} s_{13}-k_{16} \operatorname{sig}^{\rho_{13}}\left(s_{13}\right)+2 \xi \omega_{n} x_{13}+\omega_{n}^{2} x_{12}+\dot{x}_{13}^{c *}\right] \\
\omega_{n}^{-2}\left[-k_{25} s_{23}-k_{26} \operatorname{sig}^{\rho_{23}}\left(s_{23}\right)+2 \xi \omega_{n} x_{23}+\omega_{n}^{2} x_{22}+\dot{x}_{23}^{c *}\right]
\end{array}\right] .
$$

We consider the situations in which the initial relative range between the missile and the target is about $6000 \mathrm{~m}$, the initial missile coordinate is $(4500,3000,-2600)$, and the target is $(0,0,0)$, the initial missile and target velocities are $V_{m}=1500 \mathrm{~m} / \mathrm{s}$ and $V_{t}=2100 \mathrm{~m} / \mathrm{s}$, respectively, the initial 


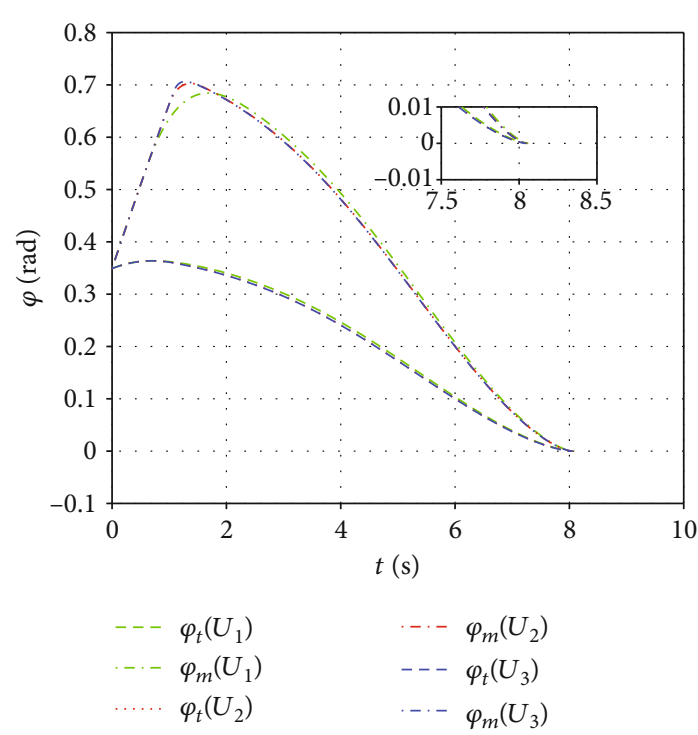

Figure 8: Direction angles $\varphi$.

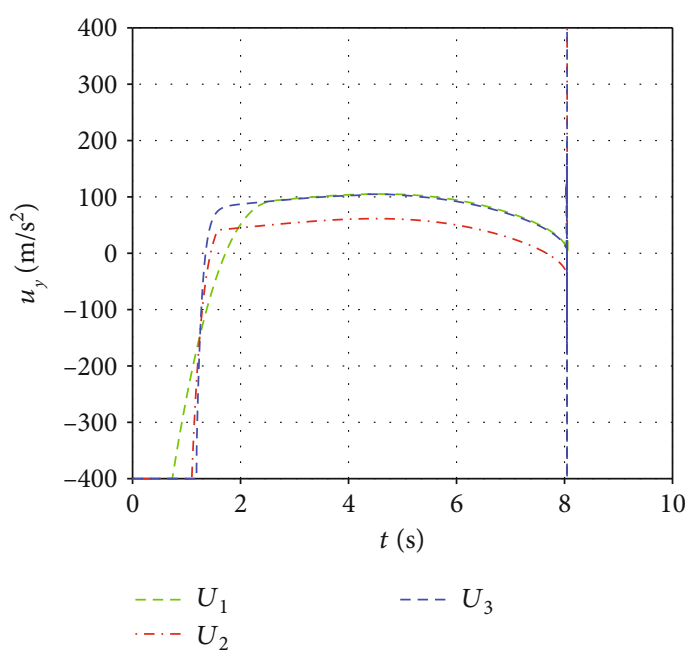

FIGURE 9: Acceleration instruction $u_{y}$.

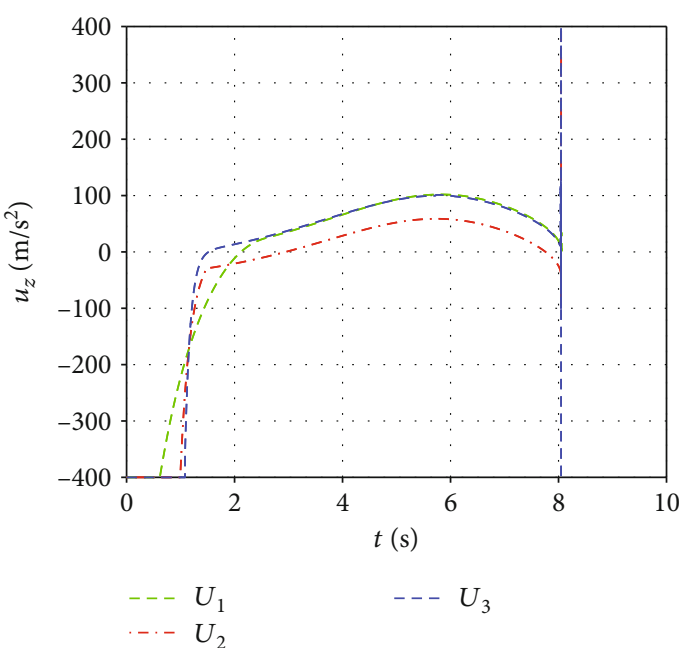

FIgURE 10: Acceleration instruction $u_{z}$.

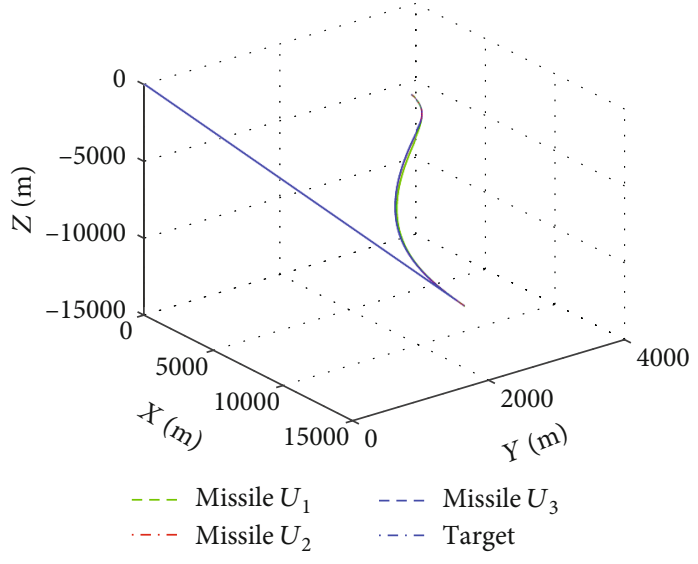

FIGURE 11: Relative trajectories.

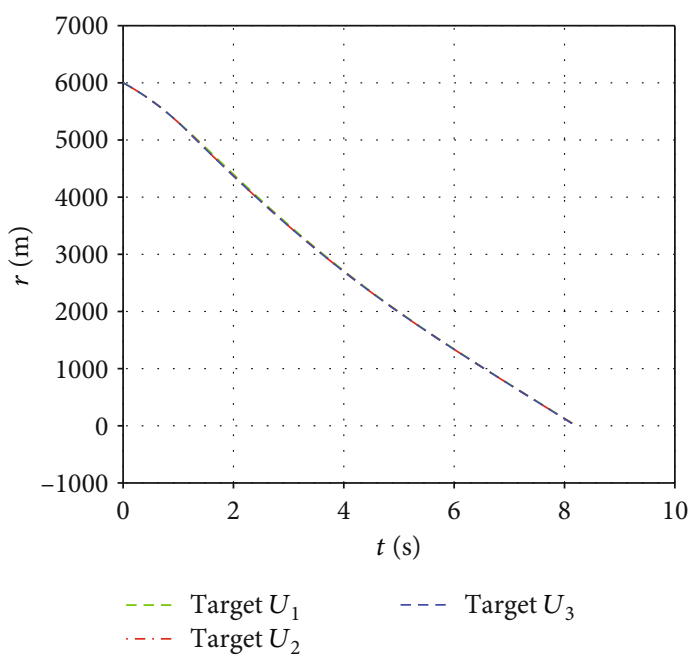

FIGURE 12: Ranges between the missile and the target.

line-of-sight angles are $\theta_{l 0}=30^{\circ}$ and $\varphi_{l 0}=30^{\circ}$, the initial missile lead angles are $\theta_{m 0}=-20^{\circ}$ and $\varphi_{m 0}=20^{\circ}$ and the target $\theta_{t 0}=-20^{\circ}$ and $\varphi_{t 0}=20^{\circ}$, the damping coefficient $\xi=0.8$, and the oscillation frequency $\omega_{n}=20$. These values are all the same in the following numerical simulation cases. According to the different target accelerations, the comparisons of guidance laws are divided into three cases.

Case 1. The target has no maneuver.

The simulation results of the three guidance laws are compared in Figures 3-10.

The simulation results show that when the target flies with no maneuver, the three guidance laws have similar results. They can make the error surfaces tend to stable states steadily, and the control input has no obvious chattering. But the convergence speeds of the error surfaces are faster under the guidance laws $U_{2}$ and $U_{3}$, which are determined by the proportion term introduced in these two guidance laws. Therefore, the guidance law designed in this paper has no clear advantage when the target flies with no maneuver. 


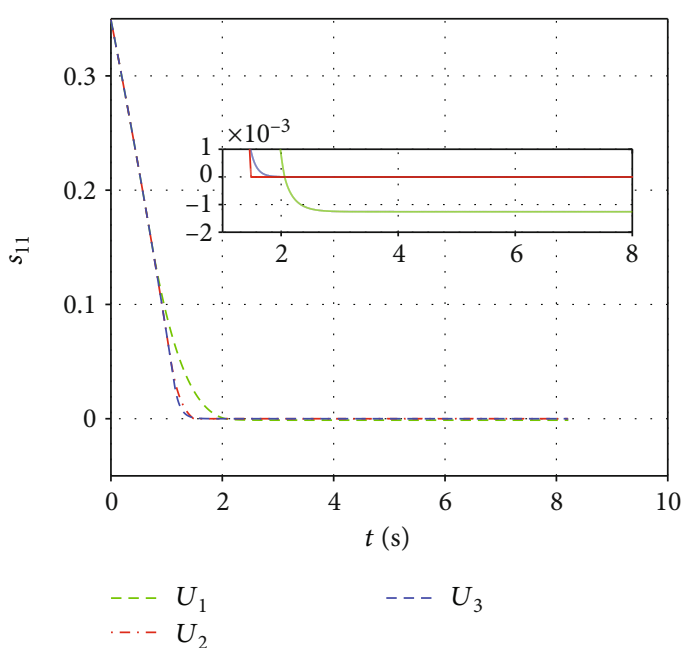

FIgURE 13: Dynamic error surfaces $s_{11}$.

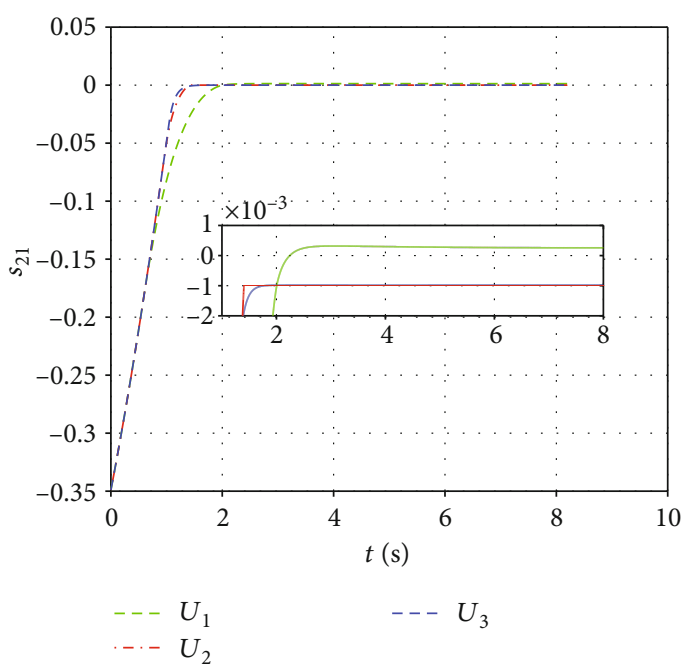

Figure 14: Dynamic error surfaces $s_{21}$.

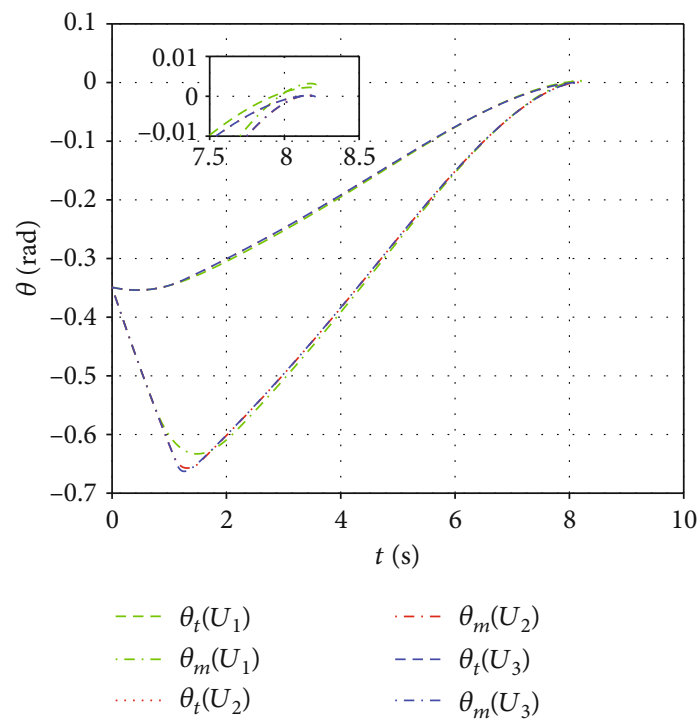

Figure 15: Direction angles $\theta$.

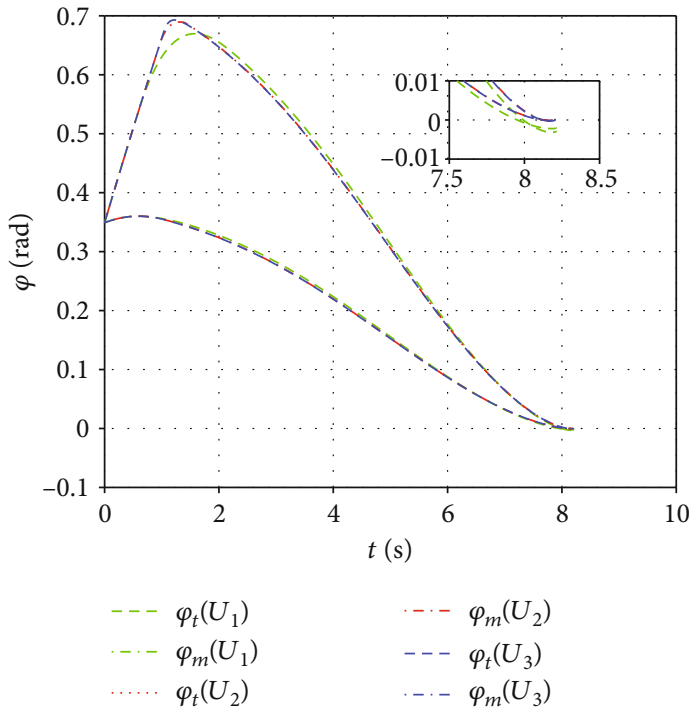

FIgURE 16: Direction angles $\varphi$.

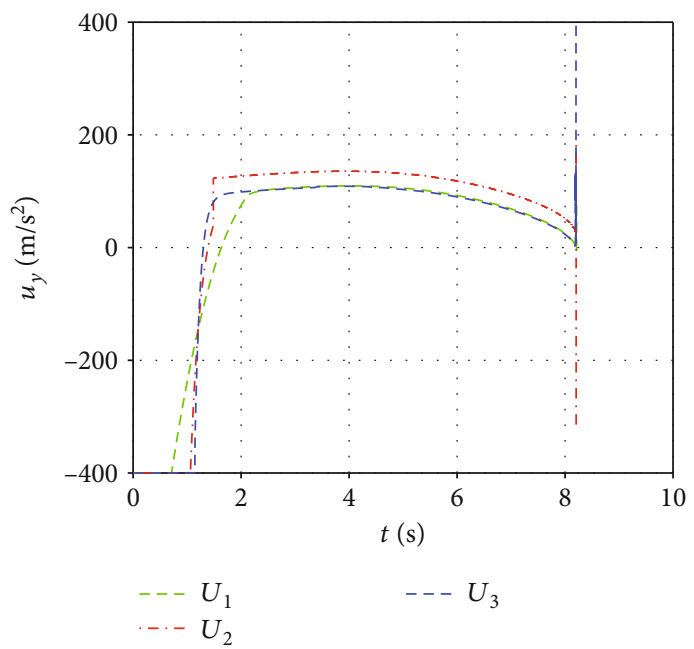

Figure 17: Acceleration instruction $u_{y}$.

Case 2. The target has small maneuvers.

(a) The target has constant maneuvers, $a_{y t}=a_{z t}=9.8$ $\mathrm{m} / \mathrm{s}^{2}$. The simulation results of the three guidance laws are compared in Figures 11-20

(b) The target maneuvers in cosine form, $a_{y t}=a_{z t}=9.8$ $\cos (\pi t / 4) \mathrm{m} / \mathrm{s}^{2}$. The simulation results of the three guidance laws are compared in Figures 21-30

The simulation results show that when the target flies with small maneuvers, there are different manifestations in some respect even though the guidance laws can complete interception missions with similar trajectories. When the target flies with small constant maneuvers, the error surfaces have steady-state errors under the guidance law $U_{1}$, which 


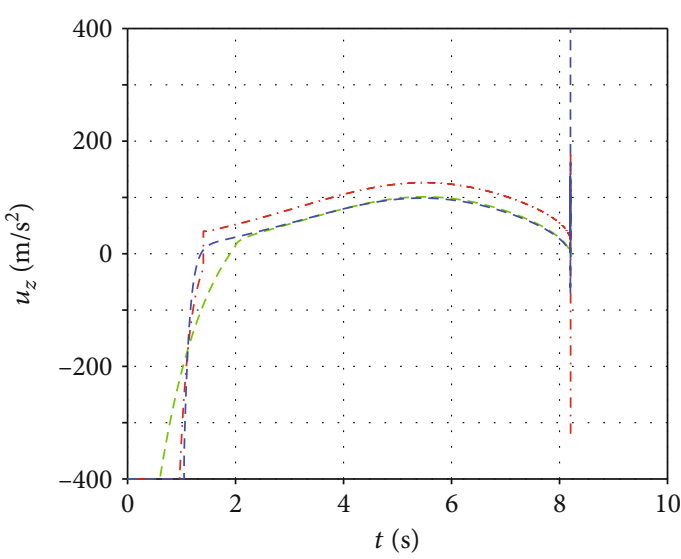

-.. $U_{1}$

-.. $U_{3}$

$\cdots U_{2}$

FIgURe 18: Acceleration instruction $u_{z}$.

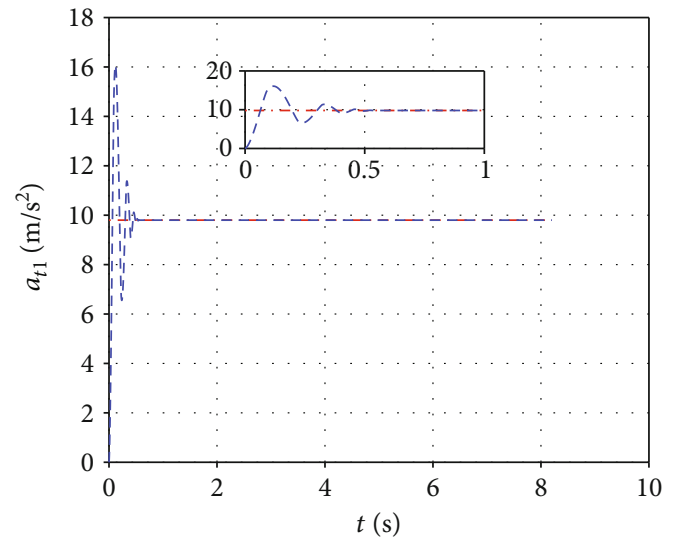

-. - Actual value of $a_{t 1}$

- - Observation of $a_{t 1}$

Figure 19: Target acceleration estimation in the pitch plane.

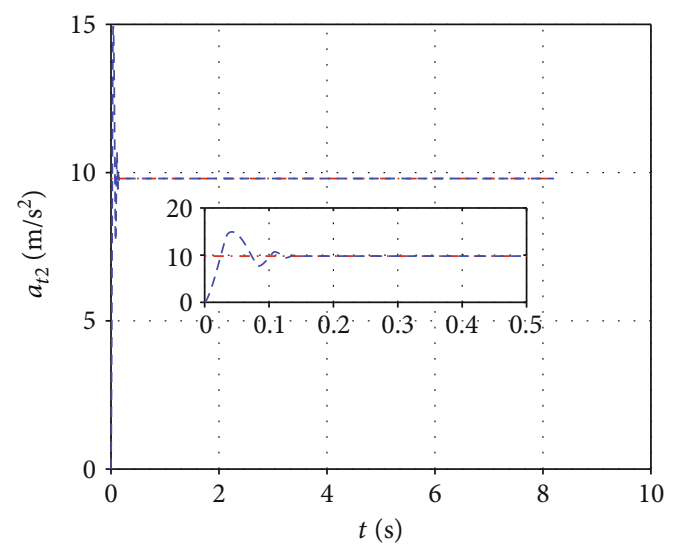

- - Actual value of $a_{t 2}$

- - Observation of $a_{t 2}$

FIgURE 20: Target acceleration estimation in the lateral plane.

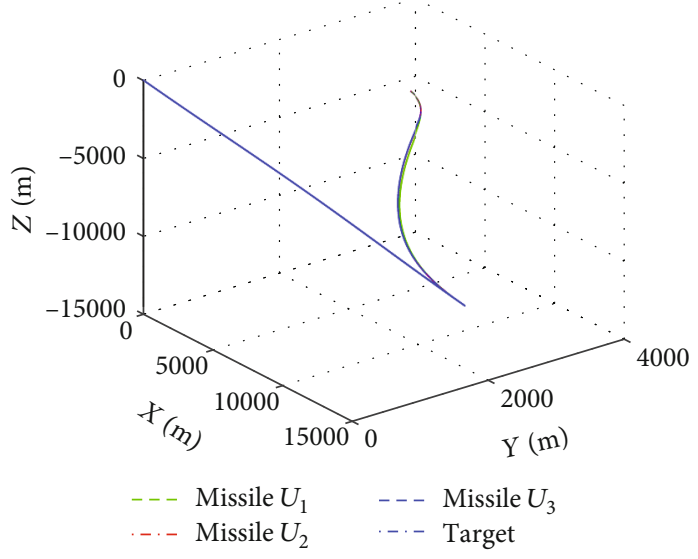

FIGURE 21: Relative trajectories.

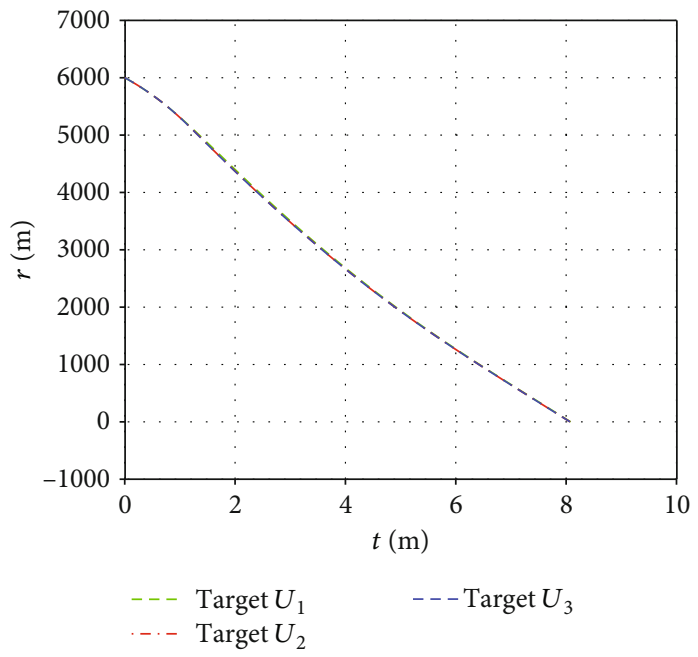

FIgURE 22: Ranges between the missile and the target.

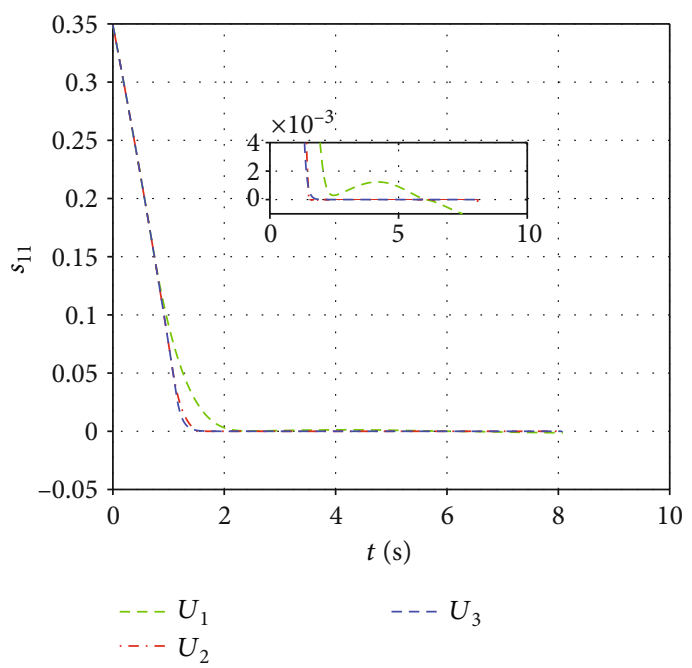

Figure 23: Dynamic error surfaces $s_{11}$. 


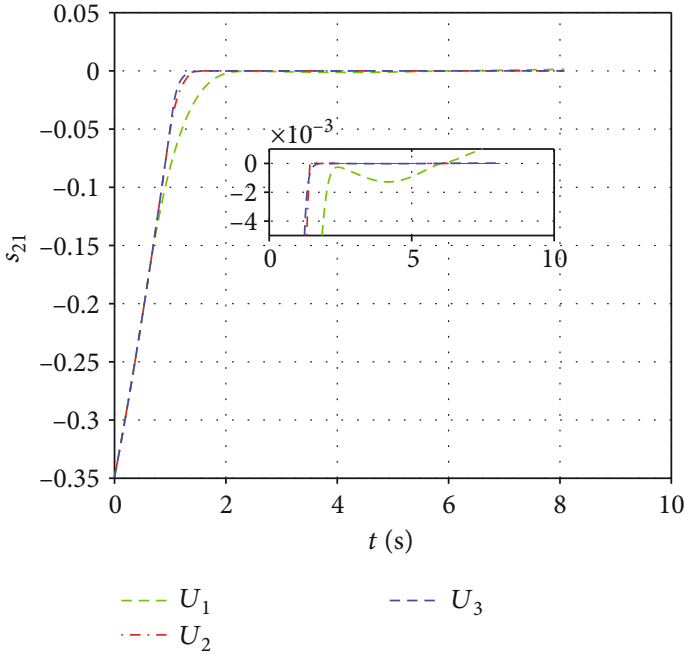

FIgURe 24: Dynamic error surfaces $s_{21}$.

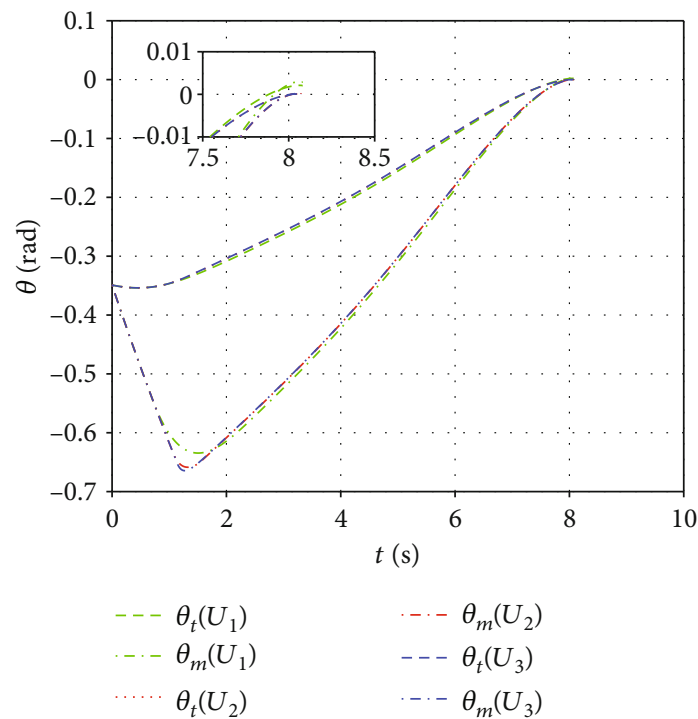

Figure 25: Direction angles $\theta$.

can be shown in Figures 13 and 14, and at the moment of collision, the missile and target have direction angles under the guidance law $U_{1}$, which can be shown in Figures 15 and 16. When the target flies with small cosine maneuvers, the error surfaces fluctuate with the peak of disturbance under guidance law $U_{1}$, which can be shown in Figures 23 and 24. And at the moment of collision, the missile and target direction angles have results similar to small constant maneuvers, which can be shown in Figures 25 and 26. Under guidance law $U_{2}$, the acceleration instructions have obvious chattering when the target flies with small constant maneuvers, which can be shown in Figures 27 and 28. The simulation results also show that there are no obvious defects in the interception process under guidance law $U_{3}$ when the target flies with small maneuvers, and the FTDO designed in this paper can track target maneuvers quickly and accurately.

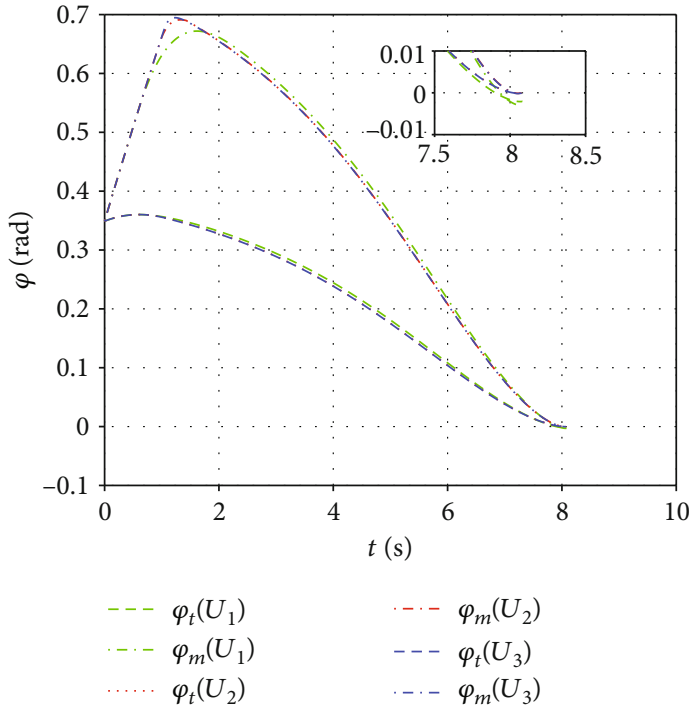

FIgURE 26: Direction angles $\varphi$.

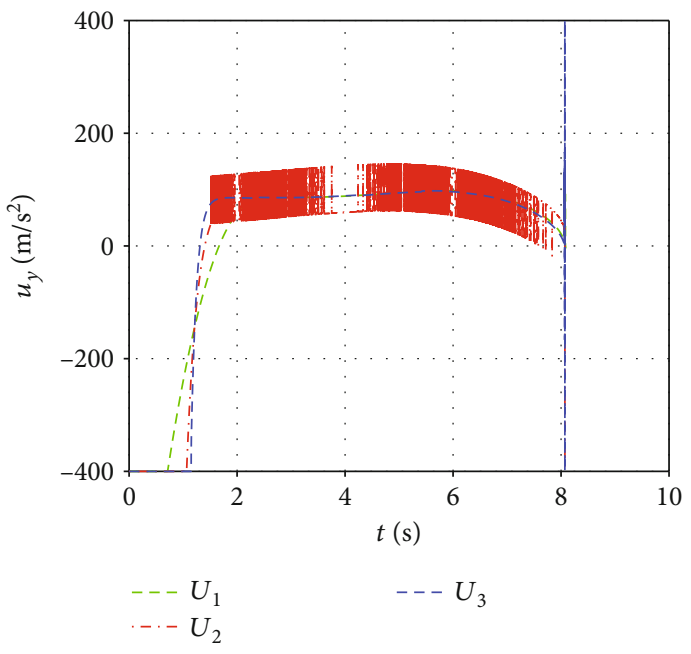

FIgURE 27: Acceleration instruction $u_{y}$.

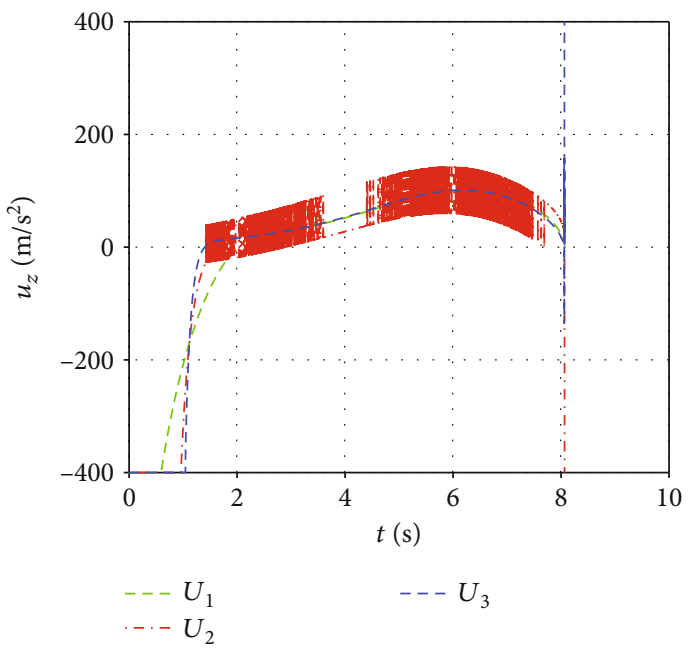

FIGURE 28: Acceleration instruction $u_{z}$. 


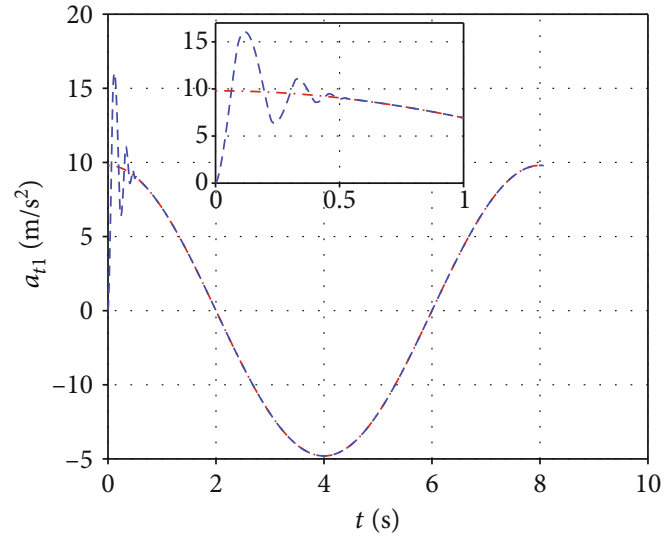

-. - Actual value of $a_{t 1}$ -- - Observation of $a_{t 1}$

Figure 29: Target acceleration estimation in the pitch plane.

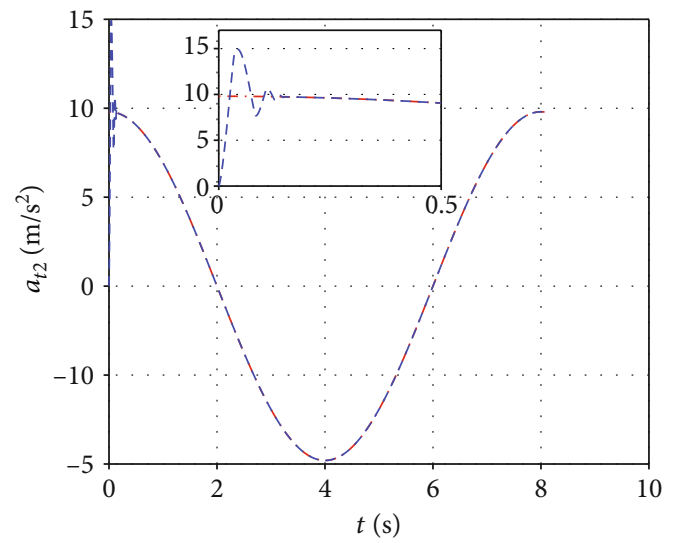

- - Actual value of $a_{t 2}$

- - Observation of $a_{t 2}$

FIgURE 30: Target acceleration estimation in the lateral plane.

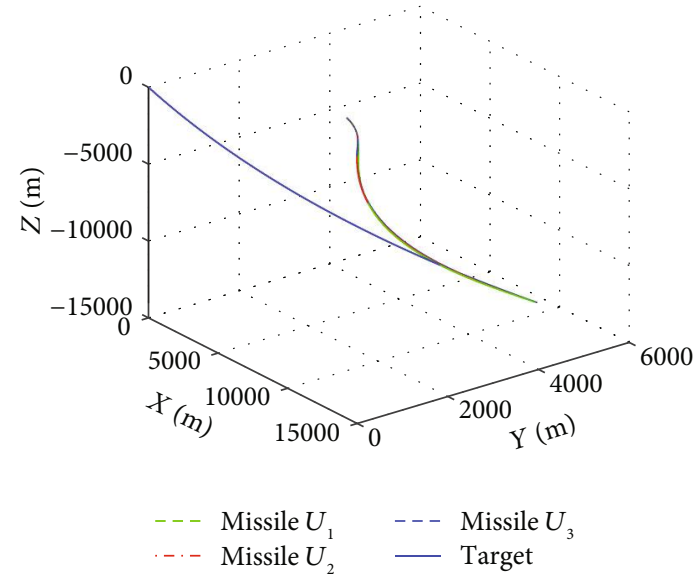

FIGURE 31: Relative trajectories.

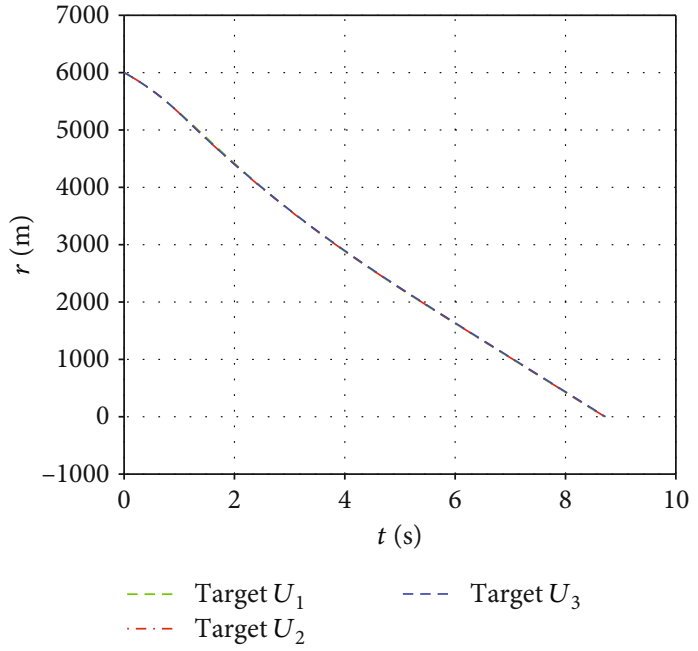

FIGURE 32: Ranges between the missile and the target.
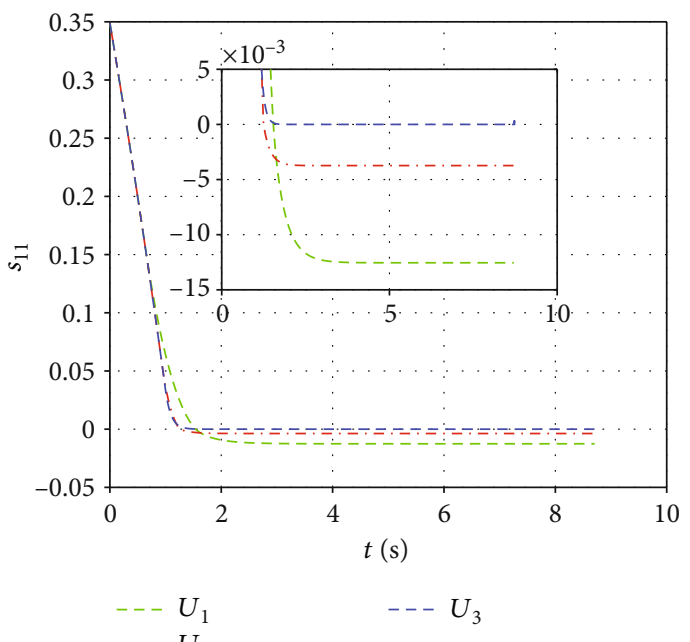

-. $U_{2}$

FIGURE 33: Dynamic error surfaces $s_{11}$.

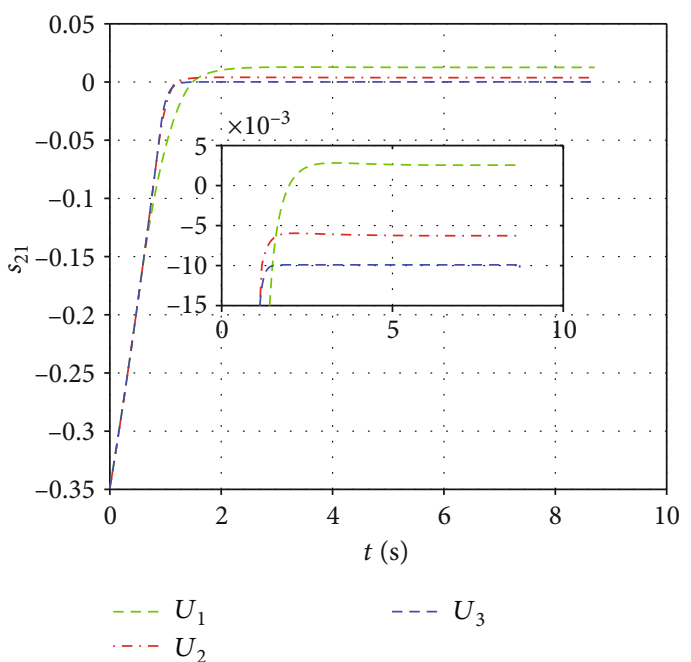

Figure 34: Dynamic error surfaces $s_{21}$. 


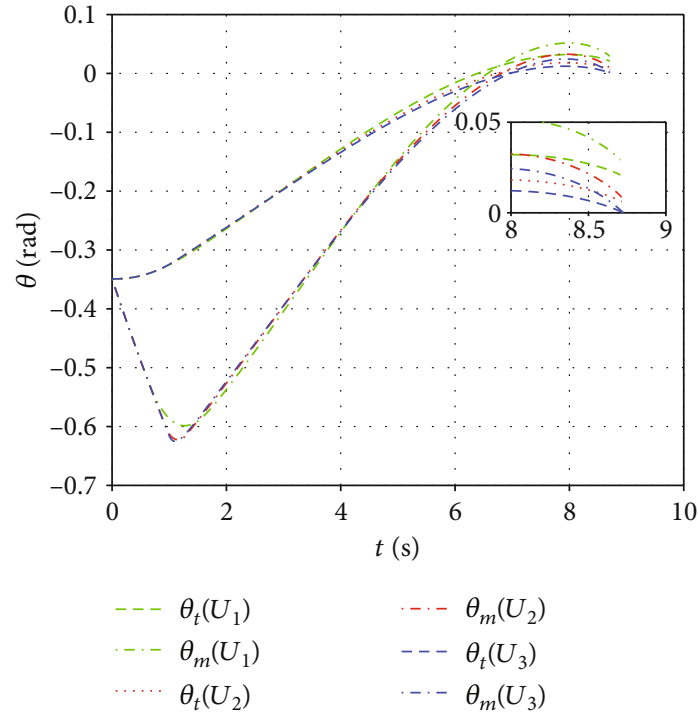

Figure 35: Direction angles $\theta$.

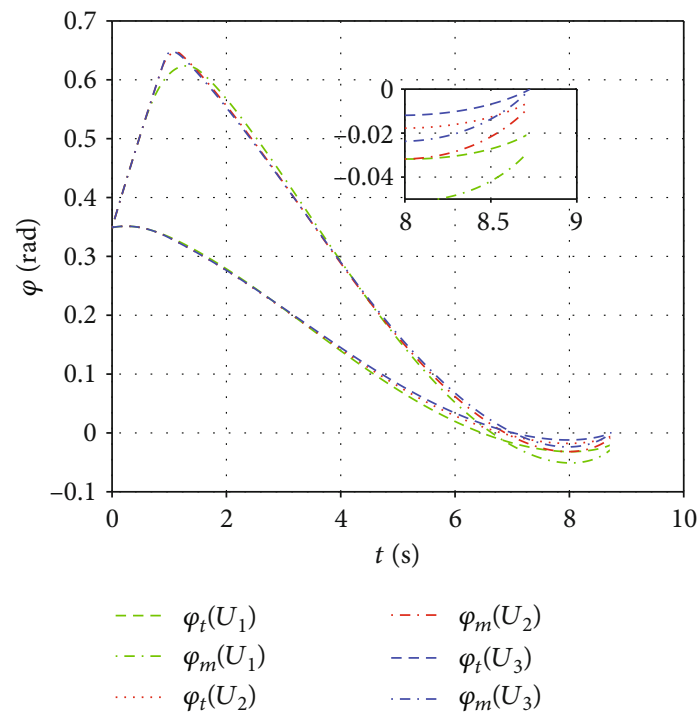

Figure 36: Direction angles $\varphi$.

Case 3. The target has large maneuvers.

(a) The target has large constant maneuvers, $a_{y t}=a_{z t}=$ $49 \mathrm{~m} / \mathrm{s}^{2}$. The simulation results of three guidance laws are compared in Figures 31-40

(b) The target maneuvers in cosine form, $a_{y t}=a_{z t}=49$ $\cos (\pi t / 4) \mathrm{m} / \mathrm{s}^{2}$. The simulation results of three guidance laws are compared in Figures 41-50

The simulation results show that missiles guided by three guidance laws, respectively, can intercept the targets when the target flies with large maneuvers, but there are significant differences in their performance. When the target flies with large constant maneuvers, the error surfaces have obvious steady-state errors under guidance laws $U_{1}$ and $U_{2}$, which can be shown in Figures 33 and 34, and at the moment of col-

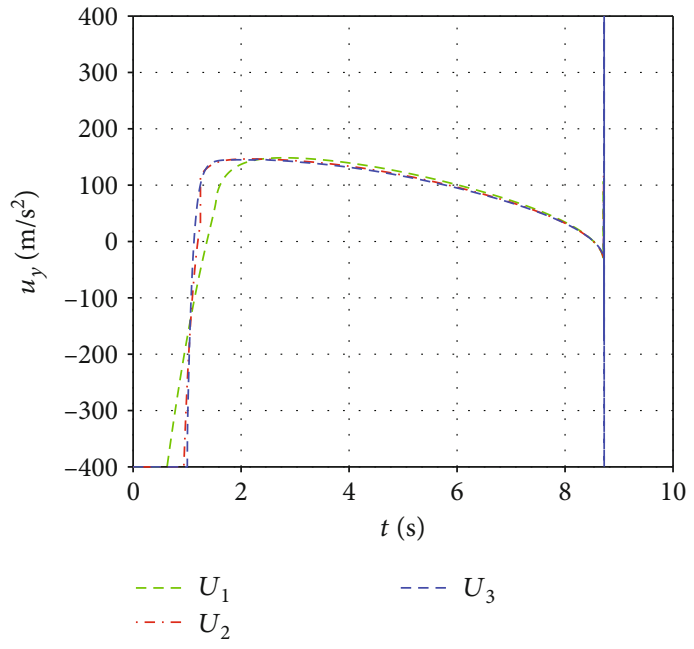

FIGURE 37: Acceleration instruction $u_{y}$.

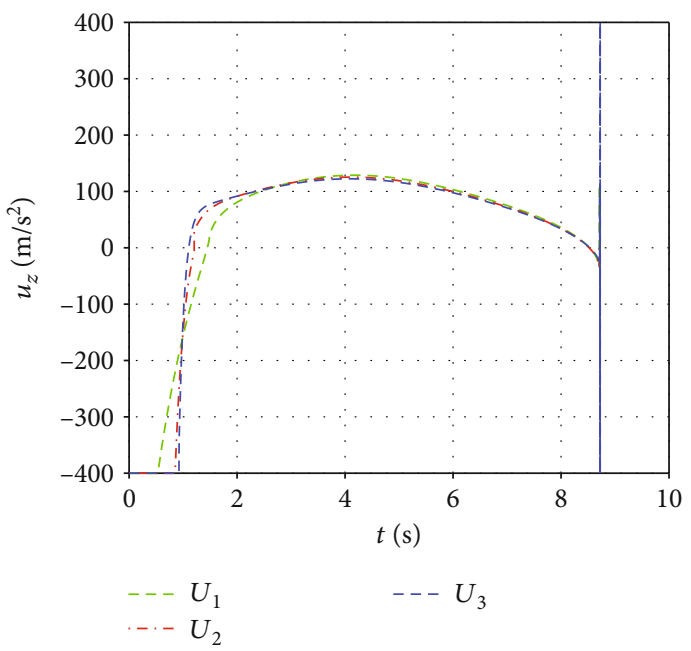

Figure 38: Acceleration instruction $u_{z}$.

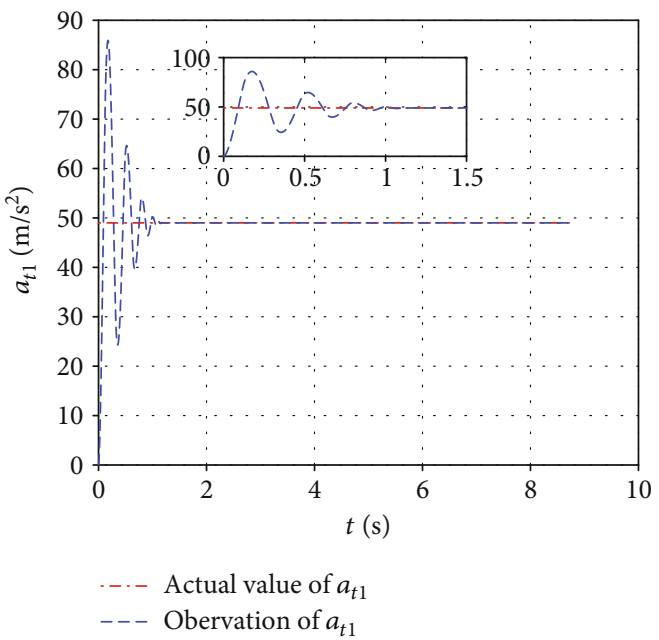

FIgURE 39: Target acceleration estimation in the pitch plane. 


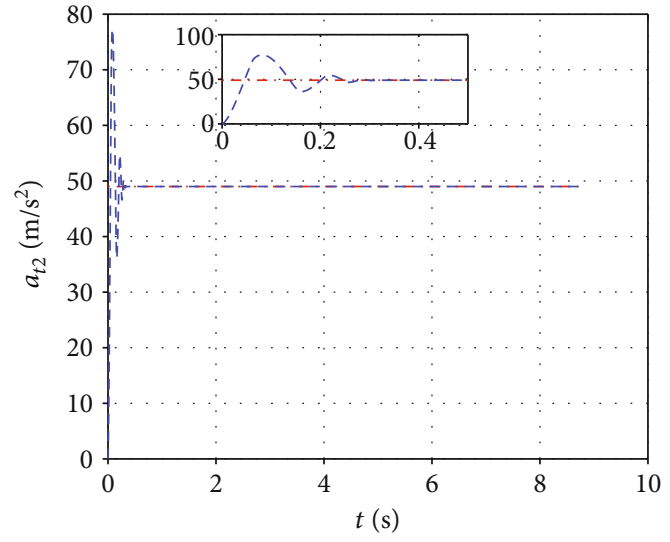

-. Actual value of $a_{t 2}$

- - Observation of $a_{t 2}$

FIgURE 40: Target acceleration estimation in the lateral plane.

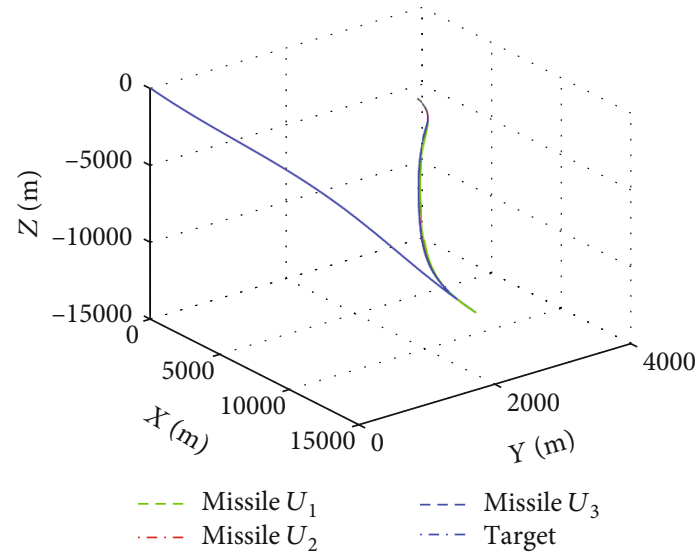

FIGURE 41: Relative trajectories.

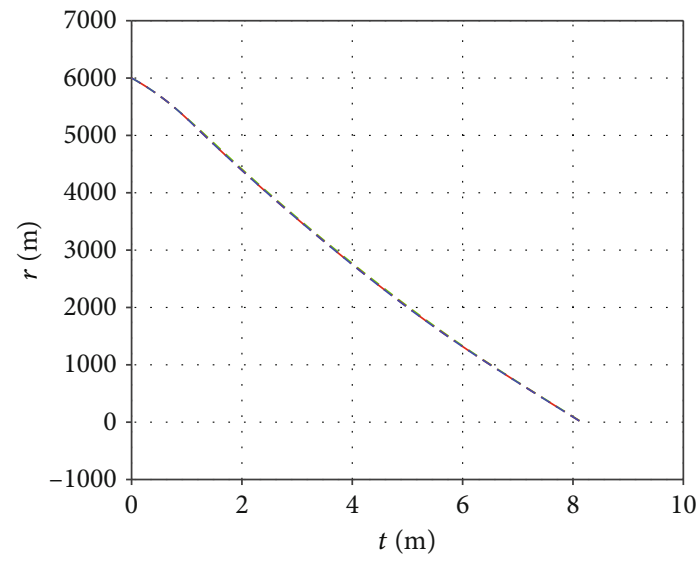

$\begin{array}{lll}\text { - } & \text { Target } U_{1} \quad \text { - }- \text { Target } U_{3} \\ \text { - . - } & \text { Target } U_{2} & \end{array}$

Figure 42: Ranges between the missile and the target.

lision, the missile and target have direction angles under guidance laws $U_{1}$ and $U_{2}$, which can be shown in Figures 35 and 36 . When the target flies with large cosine maneuvers, the error surfaces fluctuate obviously with the

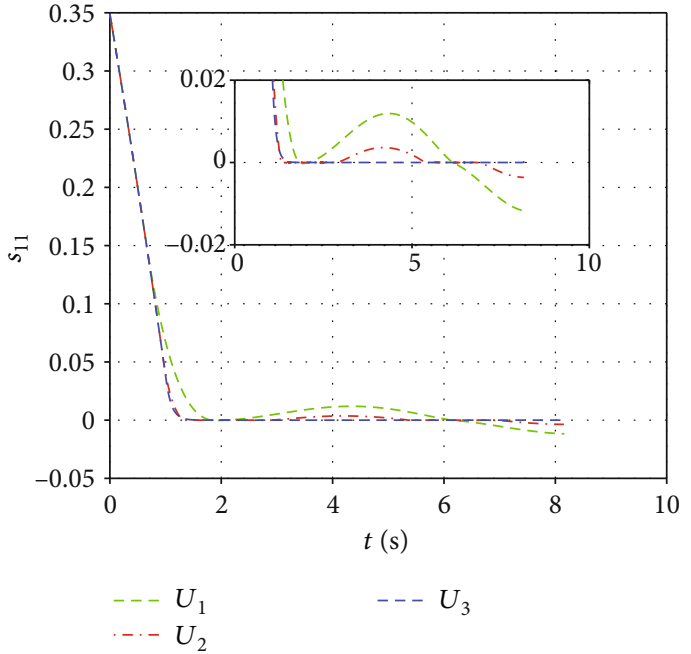

Figure 43: Dynamic error surfaces $s_{11}$.

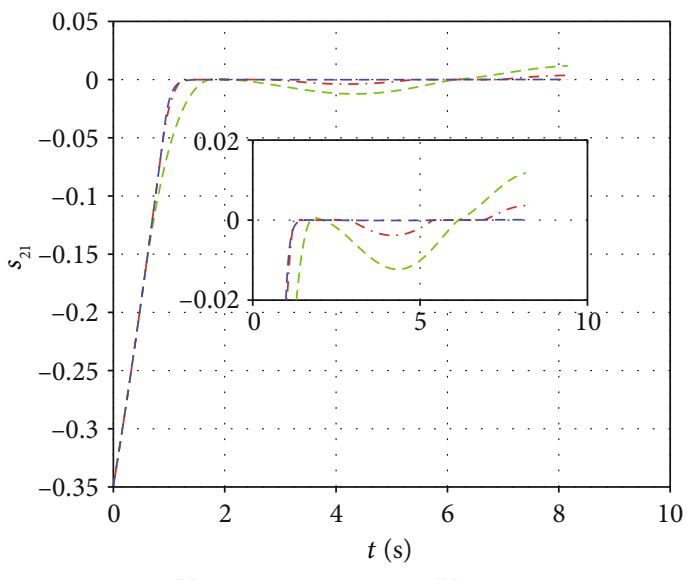

$\begin{array}{ll}---U_{1} \\ \cdots- & U_{2}\end{array}$

FIGURE 44: Dynamic error surfaces $s_{21}$.

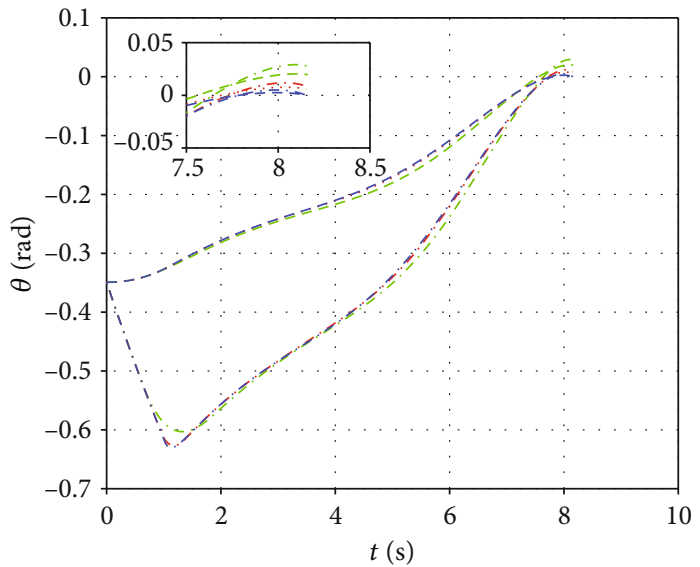

$\begin{array}{llll}-\cdots & \theta_{t}\left(U_{1}\right) & \cdots & \theta_{m}\left(U_{2}\right) \\ \cdots & \theta_{m}\left(U_{1}\right) & -\cdots & \theta_{t}\left(U_{3}\right) \\ \ldots \ldots & \theta_{t}\left(U_{2}\right) & \cdots & \theta_{m}\left(U_{3}\right)\end{array}$

Figure 45: Direction angles $\theta$. 


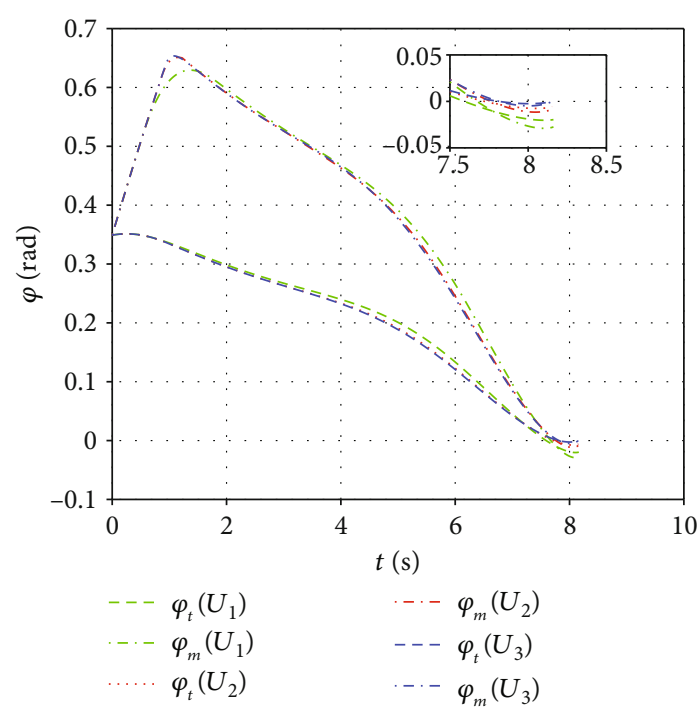

FIgURE 46: Direction angles $\varphi$.

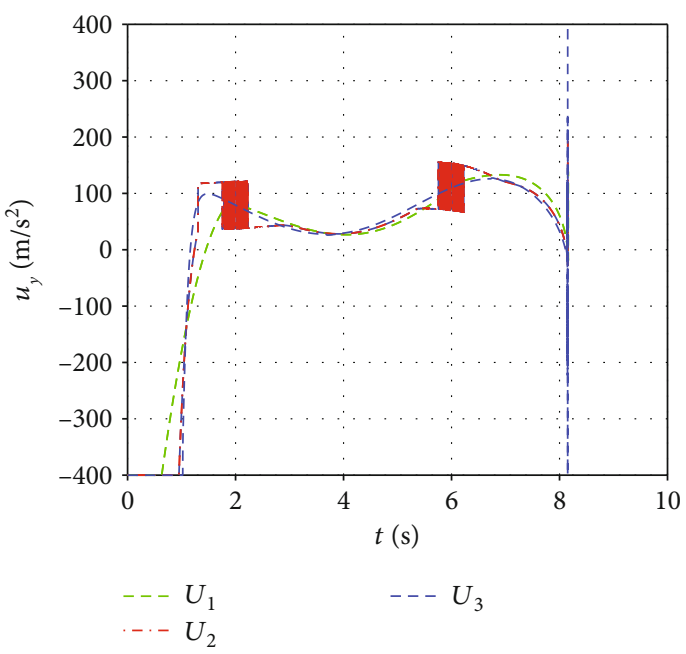

FIGURE 47: Acceleration instruction $u_{y}$.

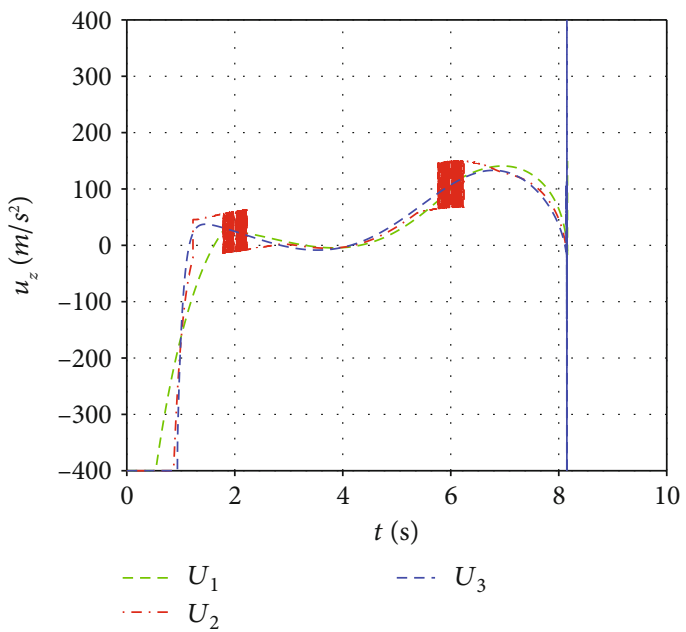

FIgURE 48: Acceleration instruction $u_{z}$.

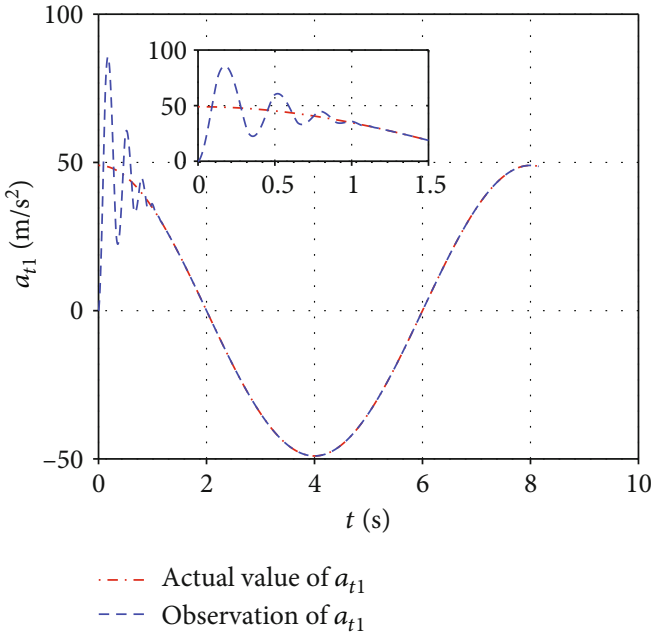

Figure 49: Target acceleration estimation in the pitch plane.

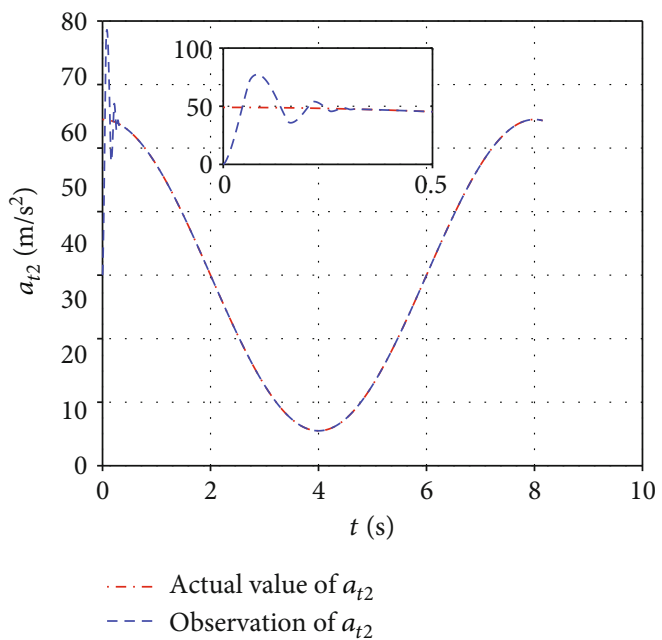

FIgURE 50: Target acceleration estimation in the lateral plane.

peak of disturbance under guidance laws $U_{1}$ and $U_{2}$, which can be shown in Figures 43 and 44. Besides, Figures 45 and 46 show that the missile and target direction angles fail to converge to zero at the moment of collision. The acceleration instructions under guidance $U_{2}$ have significant chattering compared to guidance laws $U_{1}$ and $U_{2}$, which can be shown in Figures 47 and 48. At the same time, the simulation results also show that there are no obvious defects in the interception process under the guidance law $U_{3}$. Therefore, the guidance law $U_{3}$ has better performances when the target flies with large maneuvers.

It can be found that there are many differences between the three guidance laws in intercepting hypersonic targets by comparing the simulation results under different conditions. The specific differences and reasons are as follows.

Under the guidance law $U_{1}$, the error surface cannot converge to zero but can only converge to a small neighborhood when the target flies with maneuvers, and this neighborhood expands gradually as the target maneuvers increase. This is 
because the guidance law $U_{1}$ adopts the power reaching law which can only ensure that the system converges to a small neighborhood if the system has an uncertain external disturbance, and at the same time, the range of the neighborhood is related to the parameters of the power reaching law and the upper bound of the external disturbance. Since the missile and target direction angles cannot converge to zero until the error plane remains zero, when the target is maneuvering, guidance law $U_{1}$ cannot meet the head-pursuit guidance conditions as shown in Equation (12), which can be solved by designing a disturbance observer to estimate the target maneuver online. Meanwhile, the convergence speed of guidance law $U_{1}$ can be solved by adding proportional terms.

Under the guidance law $U_{2}$, although the error surface has a fast convergence speed, the control input has a significant chattering when the target is a maneuvering flight with acceleration for the cosine. In addition, the error surface cannot converge to zero when the amplitude of the cosine maneuver is large. This is because the guidance law $U_{2}$ adopts the exponential approach law, in which the coefficient of the sign function determines the chattering and robustness of the system, and this coefficient needs to match the external disturbance. If the coefficient is relatively large, the chattering of the system will increase, while if the coefficient is relatively small, the system will be unstable. When the target maneuver is small and the coefficient of the sign function is appropriate, the error surface can quickly converge to zero. When the amplitude of the cosine maneuver is large and the coefficient of the sign function is relatively small, the system will be unstable. This problem can be solved by adjusting the symbol function coefficients online or estimating the uncertainty disturbance online and compensating for it.

\section{Conclusion}

In view of the interception problems of hypersonic targets, this paper establishes the 3D head-pursuit model. Then, a head-pursuit guidance law based on the backstepping sliding model control theory and FTDO is proposed considering the autopilot dynamic characteristics, and its stability is proved using the Lyapunov stability theorem. Finally, the guidance law proposed in this paper is compared with other guidance laws in different scenes.

We can get the following conclusions by comparing the simulation results in three cases: the guidance law proposed in this paper has better performance in the interception of hypersonic targets, especially in the face of targets with high maneuverability. This is because the guidance law proposed in this paper not only considers the convergence speed and chattering requirements of the error surface comprehensively but also combines the proportional term and the power term to accelerate the convergence speed of the error surface and weaken the buffeting. In addition, considering the characteristics of the target's large maneuver capability, the FTDO is introduced to estimate and compensate the target's maneuver online so that the interceptor missile can complete the interception according to the head-pursuit guidance conditions strictly even if the target has a large maneuver. Besides, the guidance law designed in this paper takes into account the dynamic characteristics of autopilot, which is closer to the actual guidance environment. Therefore, the effectiveness and superiorities of this head-pursuit guidance law are verified.

\section{Data Availability}

The data used to support the findings of this study are included within the article.

\section{Conflicts of Interest}

The authors declared no potential conflicts of interest with respect to the research, authorship, and/or publication of this article.

\section{Acknowledgments}

This work is supported by the Aeronautical Science Foundation of China (20170112013).

\section{References}

[1] W.-Q. Wang, "Near-space vehicle-borne SAR with reflector antenna for high-resolution and wide-swath remote sensing," IEEE Transactions on Geoscience and Remote Sensing, vol. 50, no. 2, pp. 338-348, 2012.

[2] C. Zhu and D. Mu, "Design of head-pursuit guidance law based on sliding mode control," IOP Conference Series: Materials Science and Engineering, vol. 563, article 042076, 2019.

[3] C. Guo and X. G. Liang, "Integrated guidance and control based on block backstepping sliding mode and dynamic control allocation," Proceedings of the Institution of Mechanical Engineers, Part G: Journal of Aerospace Engineering, vol. 229, no. 9, pp. 1559-1574, 2014.

[4] O. M. Golan and T. Shima, "Head pursuit guidance for hypervelocity interception," in AIAA Guidance, Navigation, and Control Conference and Exhibit, pp. 1-12, Providence, Rhode Island, 2004.

[5] O. M. Golan and T. Shima, "Precursor interceptor guidance using the sliding mode approach," in AIAA Guidance, Navigation and Control Conference, pp. 1264-1278, San Francisco, California, 2005.

[6] Z. H. Zhao, Y. Shen, and H. Liu, "A head pursuit guidance scheme based on variable structure control," Journal of Astronautics, vol. 28, no. 4, pp. 835-839, 2007.

[7] J. Jia, Y. Li, C. Chen, and Y. Y. Zhou, "Head pursuit interception sliding mode guidance law based on zero miss-distance," Aerospace Control, vol. 31, no. 1, pp. 27-31, 2013.

[8] K. Xiao, B. Sun, W. Zhang, and Y. Cai, "Head pursuit optimal adaptive sliding mode guidance law," IFAC Proceedings Volumes, vol. 46, no. 13, pp. 508-513, 2013.

[9] G. Lianzheng, S. Yi, G. Yunfeng, and Z. Lijun, "Head pursuit variable structure guidance law for three-dimensional space interception," Chinese Journal of Aeronautics, vol. 21, no. 3, pp. 247-251, 2008.

[10] L. G. Ge, Y. Shen, and L. H. Yuan, "Three-dimensional guidance model and guidance law design for head pursuit interception," Systems Engineering and Electronics, vol. 30, no. 6, pp. 1118-1121, 2008. 
[11] L. G. Ge and Y. Shen, Study of Variable Structure Guidance Law for Interceptor Using Head Pursuit Interception, Harbin Institute of Technology, 2009.

[12] S. I. Yujie and S. Song, "Three-dimensional adaptive finitetime guidance law for intercepting maneuvering targets," Chinese Journal of Aeronautics, vol. 30, no. 6, pp. 1985-2003, 2017.

[13] Y. A. Zhang, H. L. Wu, and Y. Liang, "Head pursuit guidance law considering dynamic characteristic of hybrid control system," Journal of Astronautics, vol. 36, no. 2, pp. 158-164, 2015.

[14] Y. A. Zhang, H. L. Wu, Y. Liang, and J. P. Zhang, "Threedimensional head pursuit guidance law considering dynamic characteristics of uncertain hybrid control system," Systems Engineering and Electronics, vol. 37, no. 6, pp. 1354-1361, 2015.

[15] P. Qu and D. Zhou, "A dimension reduction observer-based guidance law accounting for dynamics of missile autopilot," Proceedings of the Institution of Mechanical Engineers, Part G: Journal of Aerospace Engineering, vol. 227, no. 7, pp. 1114-1121, 2012.

[16] S. Yu, X. Yu, B. Shirinzadeh, and Z. Man, "Continuous finitetime control for robotic manipulators with terminal sliding mode," Automatica, vol. 41, no. 11, pp. 1957-1964, 2005.

[17] H. B. Zhou, Study on Guidance Law and Cooperative Guidance for Multi-Missiles Based on Finite-Time and Sliding Mode Theory, Harbin Institute of Technology, 2015.

[18] X. Liu and X. G. Liang, "Integrated guidance and control of multiple interceptor missiles based on improved distributed cooperative control strategy," Journal of Aerospace Technology and Management, vol. 11, no. 1, pp. 2119-2134, 2019. 


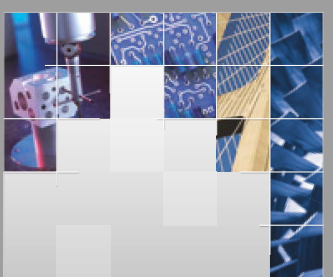

\section{Enfincering}
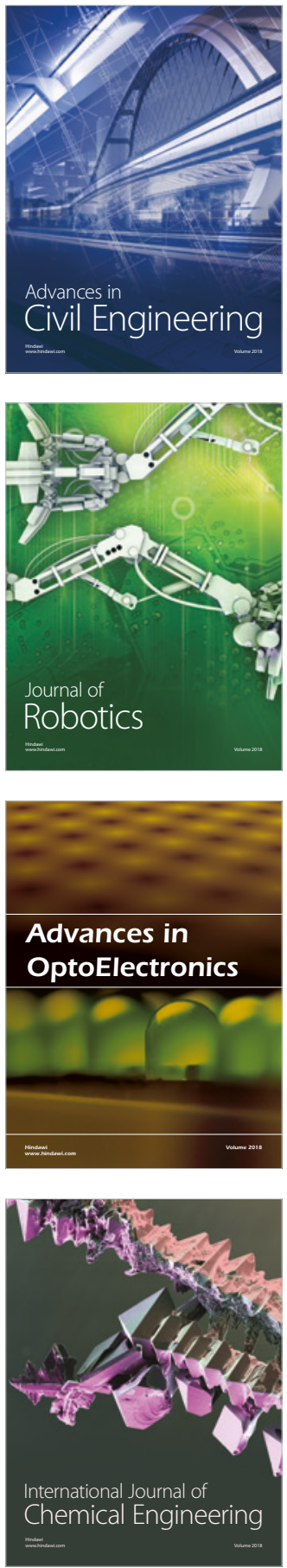

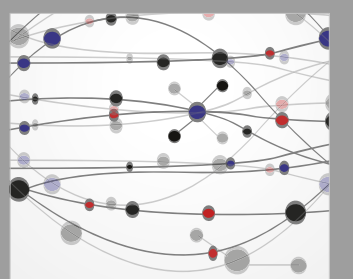

\section{Rotating \\ Machinery}

The Scientific World Journal

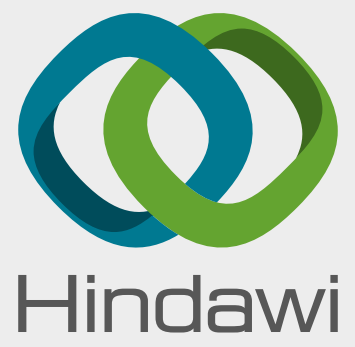

Submit your manuscripts at

www.hindawi.com
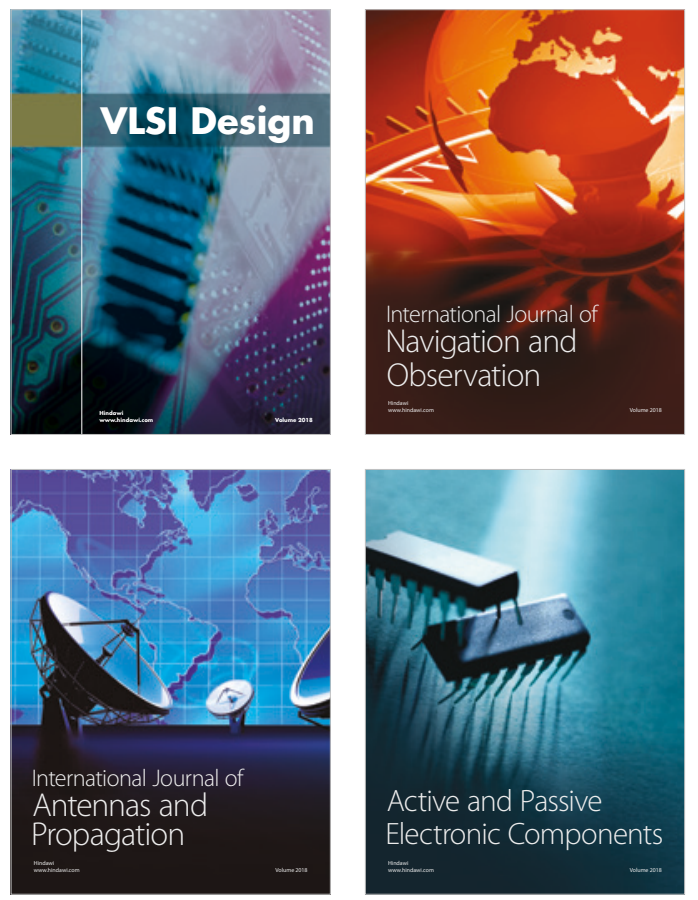
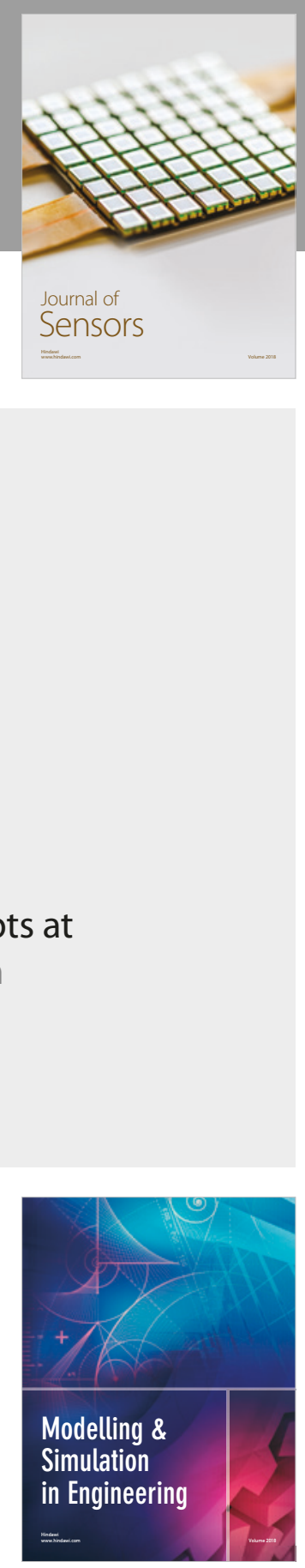

\section{Advances \\ Multimedia}
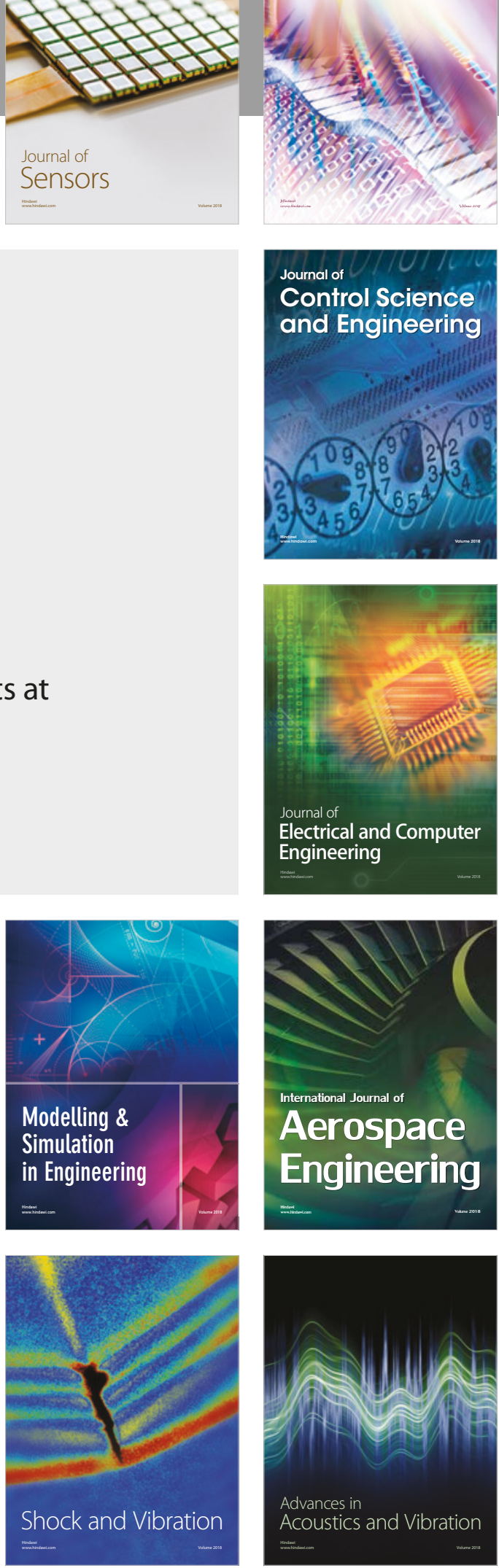